\title{
A LEVEL SET METHOD FOR THREE-DIMENSIONAL PARAXIAL GEOMETRICAL OPTICS WITH MULTIPLE POINT SOURCES *
}

\author{
SHINGYU LEUNG ${ }^{\dagger}$, JIANLIANG QIAN $\ddagger$, AND STANLEY OSHER $\S$
}

\begin{abstract}
We apply the level set method to compute the three dimensional multivalued geometrical optics term in a paraxial formulation. The paraxial formulation is obtained from the 3 -D stationary eikonal equation by using one of the spatial directions as the artificial evolution direction. The advection velocity field used to move level sets is obtained by the method of characteristics; therefore the motion of level sets is defined in phase space. The multivalued travel-time and amplitude-related quantity are obtained from solving advection equations with source terms. We derive an amplitude formula in a reduced phase space which is very convenient to use in the level set framework. By using a semi-Lagrangian method in the paraxial formulation, the method has $O\left(N^{2}\right)$ rather than $O\left(N^{4}\right)$ memory storage requirement for up to $O\left(N^{2}\right)$ multiple point sources in the five dimensional phase space, where $N$ is the number of mesh points along one direction. Although the computational complexity is still $O\left(M N^{4}\right)$, where $M$ is the number of steps in the ODE solver for the semi-Lagrangian scheme, this disadvantage is largely overcome by the fact that up to $O\left(N^{2}\right)$ multiple point sources can be treated simultaneously. Three dimensional numerical examples demonstrate the efficiency and accuracy of the method.
\end{abstract}

Key words. Hamilton-Jacobi, paraxial geometrical optics, level set method, semi-Lagrangian method

AMS Classification number: 65M25, 86A15

\section{Introduction}

Consider the geometrical optics (high frequency) approximation for 3-D acoustic wave equations. According to the Debye procedure, the leading order term defining the geometrical optics term consists of two functions, one being the eikonal satisfying the eikonal equation,

$$
|\nabla \tau|=\frac{1}{c}
$$

and the other being the amplitude solving the transport equation,

$$
\nabla \cdot\left(A^{2} \nabla \tau\right)=0
$$

where $\tau$ and $A$ are the eikonal, a.k.a., traveltime in many applications, and the amplitude function respectively; $c$ is the given wave velocity in an acoustic medium. These two equations appear in a variety of applications: high resolution seismic imaging $[15,4,25,31]$, underwater acoustics, semi-classical limit for quantum mechanics [23], and optical instruments, to name just a few.

As a first order scalar nonlinear PDE, equation (1.1) usually does not admit a global, smooth solution in physical space. By viewing the gradient components as independent functions of some parameter, for a given non-characteristic boundary condition the method of characteristics yields a smooth solution for equation (1.1) in phase space; but once projected to physical space, the solution usually is multivalued. The concept of viscosity solution singles out a unique, physically relevant weak solution among these multivalued branches of solutions, so that a continuous, global Jin.

${ }^{*}$ Received: July 6, 2004; accepted (in revised version): November 8, 2004. Communicated by Shi

${ }^{\dagger}$ Department of Mathematics, UCLA, Los Angeles, CA 90095-1555 (syleung@math.ucla.edu).

${ }^{\ddagger}$ Department of Mathematics, UCLA, Los Angeles, CA 90095-1555 (qian@math.ucla.edu).

${ }^{\S}$ Department of Mathematics, UCLA, Los Angeles, CA 90095-1555 (sjo@math.ucla.edu). 
solution is well defined in this class. However, in many applications, the multivalued solution is necessary and also physically relevant. In seismics, the later arrival travel-times which do not correspond to the viscosity solution of the eikonal equation may carry more significant energy than the viscosity solution-based first arrivals do $[12,25]$.

Naturally, one may use the method of characteristics to derive a set of ODEs to compute travel-times and amplitudes in phase space, which essentially yields everything. However, the major disadvantage of the approach is that it lacks control of resolution of the solution in physical space. Certainly, the shortcoming can be overcome to some extent by extra bookkeeping of data structures [41]. On the other hand, one may look for a PDE framework to compute these multivalued solutions in phase space; this results in the so-called Eulerian geometrical optics [3, 8]. In the past decade, there have been a lot of efforts in this direction: domain decomposition along caustic curves [2], the slowness matching method [38, 39], methods based on kinetic formulation [13, 16, 14], dynamical surface extension methods [32, 34], and Liouville equation based methods, such as the segment projection method [9], the vector-valued level set method $[26,28,18,5,6,30,29,17]$, the method based on escape parameters [11].

Extend the travel-time function $\tau=\tau(x, y, z)$ defined in a bounded spatial domain $\Omega$ to $T=T(x, y, z, \theta, \phi)$ defined in a reduced phase space via the slowness vector, equation (2.6). Consider its $t$-wavefront in the reduced phase space:

$$
T(x, y, z, \theta, \psi)=t
$$

which consists of all the wavefronts starting from all the point sources on the boundary $\partial \Omega$ and all the take-off directions pointing into the domain $\Omega$ and reaching the spatial location $(x, y, z)$ with arrival angles $(\theta, \psi)$. Here, by a point source problem for $(1.1)$ and (1.2) in the physical space, we mean that rays emit from that point in all directions with zero initial travel-times and prescribed amplitudes [20].

Now differentiate this identity w.r.t. $t$ and use the ray tracing system (2.7),

$$
\mathbf{w} \cdot \nabla_{x, y, z, \theta, \psi} T=1,
$$

where $\mathbf{w}$ denotes the right hand side of the system (2.7).

As pointed out in [5], the main issue is to identify those $(\theta, \psi)$ such that the slowness vector satisfies

$$
\mathbf{p}(\theta, \psi)=\nabla_{x, y, z} T
$$

then the eikonal equation is satisfied locally.

In the slowness matching method developed in [38, 39], this condition is enforced by solving many point source problems, equation (2.13), directly in physical space, which amounts to constructing many local fundamental solutions for the eikonal equation and identifying those slownesses satisfying (1.5) by the slowness matching condition. This method is highly efficient if travel-times from multiple point sources are desired as argued carefully in [39]. In fact it is the only method so far that stays in the physical space and at the same time resolves multivalued solutions.

To use the Liouville equation based phase space formulation more efficiently, we developed level set methods for the two-dimensional paraxial multivalued geometrical optics in $[30,29]$. In this work, we continue to develop efficient level set methods for three dimensional multivalued geometrical optics in a paraxial formulation. In this 
case, the full Liouville equation is five dimensional; by using the paraxial assumption, we essentially reduce the problem by one dimension and equation (1.4) becomes

$$
T_{z}+\mathbf{u} \cdot \nabla_{x, y, \theta, \psi} T=\frac{1}{c \sin \theta \cos \psi} ;
$$

see Section 3.2 for further details. Our framework provides multivalued geometrical optics terms for multiple point sources simultaneously in that we are able to make use of the information from not only the zero level set but also all the nonzero level sets. By using a global semi-Lagrangian method to solve level set equations, the physical space variables are directly linked to the phase variables so that the condition (1.5) can be resolved efficiently and the computational memory requirement is reduced from $O\left(N^{4}\right)$ to $O\left(N^{2}\right)$ for up to $O\left(N^{2}\right)$ multiple point sources, where $N$ is the number of mesh points along one direction. In comparison to the usual finite difference discretization of the level set equations which requires $O\left(N^{4}\right)$ memory storage, this savings is very significant in the computational space of five dimensions. Although the method proposed here has the computational complexity $O\left(M N^{4}\right)$, where $M$ is the number of steps in the ODE solver for the semi-Lagrangian scheme, it can handle up to $O\left(N^{2}\right)$ multiple point sources simultaneously without increasing computational complexity; therefore, overall it is still very efficient if the geometrical optics terms for multiple point sources are needed as in seismics [39].

In the method based on escape parameters [11], to identify those $(\theta, \psi)$ such that the condition (1.5) holds, the authors made use of the fact that the point source locations and takeoff angles are constant along the rays; therefore, five Liouville equations are used to advect these constants as tags for the rays so that the travel-time $T$ at $(x, y, z, \theta, \psi)$ from different point sources can be distinguished by checking the tags at $(x, y, z, \theta, \psi)$. Then post-processing is used to solve for $(\theta, \psi)$ satisfying $(1.5)$ at $(x, y, z)$ for specified point source locations. This approach is efficient if travel-times from up to $O\left(N^{2}\right)$ multiple point sources are desired by using a similar argument as in [39]. In terms of the paraxial formulation (1.6), the computational complexity and memory requirement of this approach are both of $O\left(N^{5}\right)$.

Our approach shares some similarities with [11], but their formulation is only for static HJ equations, and ours can be viewed for "artificial time" dependent HJ equations in terms of $z$-dependent paraxial formulation. In [11], the Liouville equations are solved by combining a local semi-Lagrangian and Dijkstra-like fast marching method, and the resulting computational memory requirement is $O\left(N^{5}\right)$ in the five dimensional reduced phase space. In this work, we explore a global semi-Lagrangian approach to solve paraxial Liouville equations so that high order ODE solvers can be used right away, and the resulting method in our setup has the advantage that the computational memory requirement is only of $O\left(N^{2}\right)$.

In the five dimensional reduced phase space, the condition (1.5) defines an object of dimension 2; therefore the object can be resolved by the intersection of zero level sets of three functions defined in $(x, y, z, \theta, \psi)$ space. This is a commonly used level set method, first proposed to compute multivalued travel-times in the high frequency asymptotics for acoustic wave equations in [26], where the multivalued travel-times are implicitly represented as self-intersecting wavefronts in the physical space. Later it was extended to compute multivalued travel-times in the high frequency asymptotics for anisotropic elastic wave equations in $[28,6]$, where the multivalued solutions for a class of steady Hamilton-Jacobi equations were computed and illustrated as self-intersecting wavefronts as well. It was also extended to compute multivalued wavefronts and 
multivalued phases in the high frequency asymptotics for the Schrödinger equation in [5], where multivalued solutions for time dependent Hamilton-Jacobi equations were constructed in a general level set framework; it was also extended to compute the multivalued gradient of the solution for time dependent and steady HamiltonJacobi equations in [18], where a level set formulation for handling the gradient of the solution was used to obtain the Liouville equation, but the formulation only yields the multivalued gradient of the solution and does not provide the multivalued solution itself. The multivalued solution was provided by adding more Liouville equations and independent variables without increasing the formal complexity in [5, 21]. In [17], the level set method is applied to computing multivalued physical observables for the semi-classical limit of the Schrödinger equation.

The advantage of following only zero level sets is that local level set methods can be designed so that the memory requirement and computational complexity are optimal to some extent $[1,27]$. The PDE based local level set method, first proposed in [27] for single level set motions and generalized in [26, 29] for vector-valued level set motions, is one of the possible approaches to reduce the computational complexity from $O\left(N^{5}\right)$ to $O\left(N^{3} \log N\right)$ in the paraxial formulation; but its memory requirement is still of $O\left(N^{4}\right)$. The tree-based local level set method also only follows the zero level set by putting more nodes near the zero level set [35, 24]; this reduces the computational complexity from $O\left(N^{5}\right)$ to $O\left(N^{3} \log N\right)$, and the memory requirement $O\left(N^{4}\right)$ to $O\left(N^{2} \log N\right)$ on average. The semi-Lagrangian method proposed in [24], which in turn is an extension of [35], is based on short time evolution of an Euler step, so that the zero level set can be tracked locally using a dyadic tree structure. Since such local level set methods follow only zero level sets, they can handle only wave propagation with a single point source.

Given multiple point sources, we can certainly initialize the level set function so that their initial zero level sets represent those multiple point sources. With this initialization, one can still get all the multi-arrival rays from multiple point sources. However, on the later times, one cannot distinguish rays from different point sources because all rays are represented by the same intersection of zero level sets essentially. To treat multiple point sources in the framework of using only zero level sets, it is possible to use the idea proposed in [5] by augmenting the reduced phase space with one more dimension such that the solutions for multiple point sources are viewed as graphs in the augmented phase space. This idea is worth exploring further.

If limited to a single point source, then our approach can also be viewed in the framework of [5]. Thus the work presented here serves as a link between the work in $[26,28,5]$ and that in $[11]$.

The rest of the paper is organized as follows: Section 2 presents the paraxial formulation for the 3-D eikonal equation; Section 3 presents the level set formulation for multivalued wavefronts, travel-times and amplitudes; Section 4 gives implementation details for the level set method; Section 5 demonstrates the accuracy of the proposed semi-Lagrangian level set method with extensive numerical examples; Section 6 concludes the paper.

\section{Three dimensional Paraxial Formulation for Eikonal Equation}

Consider a point source condition for the 3-D eikonal equation defined in an open, bounded domain $\Omega \subset \mathbf{R}^{3}$. To emphasize the point source condition the eikonal equation is rewritten as follows,

$$
\left|\nabla_{\mathbf{x}} \tau\left(\mathbf{x}, \mathbf{x}_{s}\right)\right|=\frac{1}{c(\mathbf{x})},
$$




$$
\lim _{\mathbf{x} \rightarrow \mathbf{x}_{s}} \frac{\tau\left(\mathbf{x}, \mathbf{x}_{s}\right)}{\left|\mathbf{x}-\mathbf{x}_{s}\right|}=\frac{1}{c\left(\mathbf{x}_{s}\right)}, \tau \geq 0
$$

where $\mathbf{x}_{s}$ is a given source point. Here by a point source, we mean that rays emit from that point in all directions with zero initial travel-times and prescribed amplitudes.

To apply the method of characteristics, we first parameterize the $3-\mathrm{D}$ unit vectors by spherical coordinates. Points on a unit sphere away from the $x$-axis can be uniquely represented by the following rotated spherical coordinates,

$$
\begin{aligned}
& x=\cos \theta, \\
& y=\sin \theta \sin \psi, \\
& z=\sin \theta \cos \psi,
\end{aligned}
$$

where $\theta \in(0, \pi)$ is the angle between the point and the positive $x$-axis, and $\psi \in[-\pi, \pi)$ is the angle between the positive $z$-axis and the projection of the point onto the $y$ - $z$ plane. Figure 2.1 shows the standard and rotated spherical coordinates. Then the slowness vector $\nabla \tau=\mathbf{p}$ can be represented as

$$
\begin{aligned}
& p_{1}=\frac{\cos \theta}{c}, \\
& p_{2}=\frac{\sin \theta \sin \psi}{c}, \\
& p_{3}=\frac{\sin \theta \cos \psi}{c} .
\end{aligned}
$$

By using the above parameterization we have the following ray tracing system

$$
\begin{aligned}
\frac{d x}{d t} & =c \cos \theta, \\
\frac{d y}{d t} & =c \sin \theta \sin \psi, \\
\frac{d z}{d t} & =c \sin \theta \cos \psi, \\
\frac{d \theta}{d t} & =\sin \theta \frac{\partial c}{\partial x}-\cos \theta\left(\cos \psi \frac{\partial c}{\partial z}+\sin \psi \frac{\partial c}{\partial y}\right), \\
\frac{d \psi}{d t} & =\frac{1}{\sin \theta}\left(\sin \psi \frac{\partial c}{\partial z}-\cos \psi \frac{\partial c}{\partial y}\right),
\end{aligned}
$$

with initial conditions

$$
\begin{gathered}
\left.x\right|_{t=0}=x_{s}, \\
\left.y\right|_{t=0}=y_{s}, \\
\left.z\right|_{t=0}=z_{s}, \\
\left.\theta\right|_{t=0}=\theta_{s}, \\
\left.\psi\right|_{t=0}=\psi_{s},
\end{gathered}
$$

where $\mathbf{x}_{s}=\left(x_{s}, y_{s}, z_{s}\right)$, and $\theta_{s}$ and $\psi_{s}$ vary from 0 to $\pi$ and from $-\pi$ to $\pi$ respectively. One can compare this formulation with the one from $[19,26]$. We use these rotated spherical coordinates rather than the standard spherical coordinates system because points on the $z$-axis can now be uniquely represented. Although points on the $x$-axis 

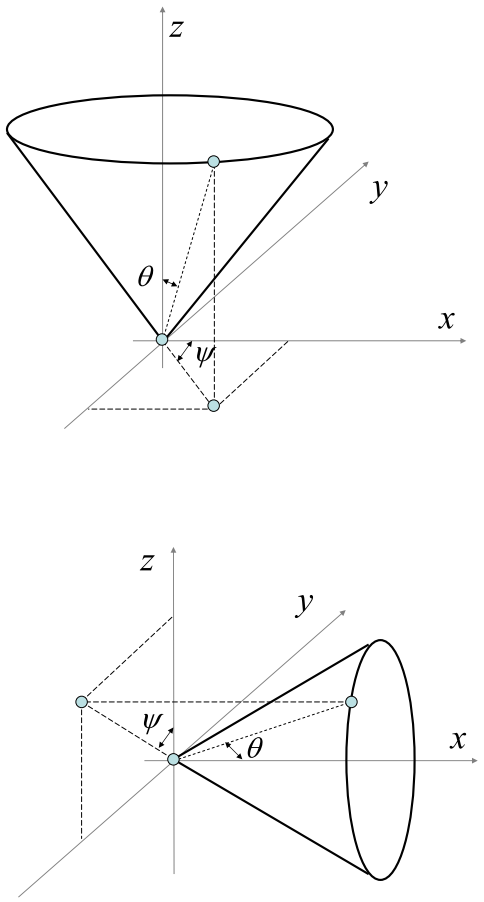

FIG. 2.1. Spherical coordinates and rotated spherical coordinates

in this rotated coordinate system may still cause problems, these points are actually out of our computational domain according to the paraxial assumption which will be discussed below.

Next we extend the arrival-time function $\tau(x, y, z)$ to the reduced phase space $\{(x, y, z, \theta, \psi)\}$, denoting it as $T(x, y, z, \theta, \psi)$, and consider the $t$-wavefront expanding from the source point:

$$
T(x, y, z, \theta, \psi)=t .
$$

Differentiating this identity with respect to $t$, we have

$$
\frac{d x}{d t} T_{x}+\frac{d y}{d t} T_{y}+\frac{d z}{d t} T_{z}+\frac{d \theta}{d t} T_{\theta}+\frac{d \psi}{d t} T_{\psi}=1
$$

with the boundary condition

$$
T\left(x_{s}, y_{s}, z_{s}, \theta_{s}, \psi_{s}\right)=0,
$$

for $0 \leq \theta_{s} \leq \pi$ and $-\pi \leq \psi_{s} \leq \pi$.

Since equation (2.10) is a linear advection equation, one may be tempted to solve it directly with the condition (2.11). However, for a given $(x, y, z) \neq\left(x_{s}, y_{s}, z_{s}\right)$, $T(x, y, z, \cdot, \cdot)$ is well defined for at least one $(\theta, \psi)$ corresponding to the first arrival, but 
it is not necessarily well defined for all $\theta$ and $\psi$; these facts imply that the solution surface $T$ in phase space is extremely singular. In other words, equations (2.10) and (2.11) are not well-posed. To obtain a well-posed problem, we will assume the paraxial condition and use a level set formulation.

In some applications, for example wave propagation in reflection seismics [7], the arrival-times of interest are carried by the so-called sub-horizontal rays $[15,37$, 31], where sub-horizontal means "oriented in the positive $z$-direction". A convenient characterization for sub-horizontal rays is that

$$
\frac{d z}{d t} \geq c \sin \theta_{\max } \cos \psi_{\max }>0
$$

for some $\theta_{\max }$ and $\psi_{\max }$ with $\pi / 2<\theta_{\max }<\pi$ and $0<\psi_{\max }<\pi / 2$.

This inequality holds for rays making angles $\theta$ and $\psi$ satisfying $|\theta| \leq \theta_{\max }<\pi$ and $|\psi| \leq \psi_{\max }<\pi / 2$. Therefore, in the paraxial formulation we are interested in only a part of the ray field. The travel-times corresponding to these sub-horizontal rays satisfy the following paraxial eikonal equation

$$
\frac{\partial \tau}{\partial z}=\sqrt{\max \left(\frac{1}{c^{2}}-\left(\frac{\partial \tau}{\partial x}\right)^{2}-\left(\frac{\partial \tau}{\partial y}\right)^{2}, \frac{\sin ^{2} \theta_{\max } \cos ^{2} \psi_{\max }}{c^{2}}\right)} .
$$

To be specific, consider

$$
\Omega=\left\{(x, y, z): x_{\min } \leq x \leq x_{\max }, y_{\min } \leq y \leq y_{\max }, 0 \leq z \leq z_{\max }\right\}
$$

and assume that the point source is located on the surface: $x_{\min } \leq x_{s} \leq x_{\max }, y_{\min } \leq$ $y \leq y_{\max }$ and $z_{s}=0$. By the sub-horizontal condition we can use depth $z$ as the running parameter so that we have the following reduced system

$$
\begin{aligned}
x_{z} & =\frac{1}{\cos \psi \tan \theta}, \\
y_{z} & =\tan \psi, \\
\theta_{z} & =\frac{c_{x}}{c \cos \psi}-\frac{c_{z}+c_{y} \tan \psi}{c \tan \theta}, \\
\psi_{z} & =\frac{c_{z} \tan \psi-c_{y}}{c \sin ^{2} \theta},
\end{aligned}
$$

where $x \in\left[x_{\min }, x_{\max }\right], y \in\left[y_{\min }, y_{\max }\right], \theta \in\left[\epsilon_{\theta}, \pi-\epsilon_{\theta}\right]$ and $\psi \in\left[\epsilon_{\psi}-\pi / 2, \pi / 2-\epsilon_{\psi}\right]$.

\section{Level Set Formulation}

3.1. Representation of a Point Source. We first assume that a single point source is located at the origin. Therefore, rays from this point source can now be represented as the intersection of zero level sets of two level set functions, $\phi^{1}(z ; x, y, \theta, \psi)$ and $\phi^{2}(z ; x, y, \theta, \psi)$.

Differentiating level sets of these functions with respect to $z$, we get the following level set equations which govern the motion of the corresponding zero level sets,

$$
\phi_{z}^{m}+\frac{d x}{d z} \phi_{x}^{m}+\frac{d y}{d z} \phi_{y}^{m}+\frac{d \theta}{d z} \phi_{\theta}^{m}+\frac{d \psi}{d z} \phi_{\psi}^{m}=0
$$

for $m=1,2$. These equations can be rewritten as

$$
\phi_{z}^{m}+\mathbf{u} \cdot \nabla_{x, y, \theta, \psi} \phi^{m}=0
$$


for $m=1,2$, where the velocity field $\mathbf{u}=\left(u^{1}, u^{2}, u^{3}, u^{4}\right)$ is given by the ray tracing system $(2.15)$.

On $z=0$, we initialize the level set functions by

$$
\phi^{1}(0 ; x, y, \theta, \psi)=x \text { and } \phi^{2}(0 ; x, y, \theta, \psi)=y,
$$

so that the intersection of zero level sets represents the location of the point source at the origin.

3.2. Arrival-time. Arrival-time can be computed by inverting the third equation in (2.7) locally. This gives

$$
T_{z}+\mathbf{u} \cdot \nabla_{x, y, \theta, \psi} T=\frac{1}{c \sin \theta \cos \psi},
$$

where $T=T(z ; x, y, \theta, \psi)$; this equation will be solved along with (3.2).

On $z=0$, we initialize the traveltime function by

$$
T(0 ; x, y, \theta, \psi)=0,
$$

so that the initial traveltime is zero.

To obtain the multivalued arrival-time on $z=z^{*}$, we first solve equations (3.2) and (3.4) up to $z=z^{*}$. We then compute the intersection of the zero level sets, denoted by

$$
\Sigma_{\mathbf{0}}=\left\{(x, y, \theta, \psi): \phi^{1}\left(z^{*} ; x, y, \theta, \psi\right)=\phi^{2}\left(z^{*} ; x, y, \theta, \psi\right)=0\right\} \subset \mathbb{R}^{4} .
$$

The arrival-times at $\left(z^{*} ; x, y\right)$ can be determined by projecting $T\left(z^{*} ; \Sigma_{\mathbf{0}}\right)$ onto the $x$-y plane.

3.3. Representation of Multiple Point Sources. However, we have noticed that the level set functions contain much more information than what we have used in the above algorithm, since not only the zero level set but also the non-zero level sets are useful. Rays emanating from a point source in phase space are not necessarily represented by the intersection of two zero level sets only. We can define rays from a point source at location $\left(x_{s}, y_{s}\right)$ by the intersection of $\left\{\phi^{1}=x_{s}\right\}$ and $\left\{\phi^{2}=y_{s}\right\}$.

Under the same velocity field, given by $\mathbf{u}$, to find all rays on $z=z^{*}$ from this point source $\left(x_{s}, y_{s}\right)$, one only needs to determine the set

$$
\Sigma_{\mathbf{x}_{s}}=\left\{(x, y, \theta, \psi): \phi^{1}\left(z^{*} ; x, y, \theta, \psi\right)-x_{s}=\phi^{2}\left(z^{*} ; x, y, \theta, \psi\right)-y_{s}=0\right\},
$$

rather than the one defined by (3.6). In other words, using the initial conditions (3.3), a point $\left(x_{i}, y_{j}, \theta_{k}, \psi_{l}\right)$ on $z=z^{*}$ can be connected to the point

$$
\left(\phi^{1}\left(z^{*} ; x_{i}, y_{j}, \theta_{k}, \psi_{l}\right), \phi^{2}\left(z^{*} ; x_{i}, y_{j}, \theta_{k}, \psi_{l}\right), \theta_{0}, \psi_{0}\right)
$$

on $z=0$ through the characteristic curve of (3.2) for some initial values $\left(\theta_{0}, \psi_{0}\right)$. More importantly, the initial conditions (3.3) can be reinterpreted as follows. All points, not only $\left(x_{i}, y_{j}\right)$, but also $\forall x \in\left[x_{\min }, x_{\max }\right]$ and $\forall y \in\left[y_{\min }, y_{\max }\right]$, can be treated as locations of point sources. Therefore, we are able to determine multi-arrival rays from multiple point sources.

The representation of point sources discussed above is of course not the only way that we can represent rays from multiple point sources. One can actually define 
$\Sigma_{\mathbf{0}}$ to denote all rays from multiple point sources. For example, $\phi^{1}(0 ; x, y, \theta, \psi)$ and $\phi^{2}(0 ; x, y, \theta, \psi)$ need to be independent of the angles $\theta$ and $\psi$, and $\left\{\phi^{1}(0 ; x, y, \theta, \psi)=\right.$ $0\} \cap\left\{\phi^{2}(0 ; x, y, \theta, \psi)=0\right\}$ at locations of those point sources. With this formulation, one can still get all the multi-arrival rays from multiple point sources. But, on the level $z=z^{*}$, one cannot distinguish rays from different point sources because all rays are represented by the same intersection of zero level sets essentially. However, using the formulation proposed here, we can separate the rays from different point sources by using only one set of level set functions and making use of all the available level sets.

3.4. Amplitude. The amplitude of the rays can also be computed using the current formulation. Defining $\tilde{A}=\tilde{A}(z ; x, y)$, we have

$$
\tilde{A}(z ; x, y)=\frac{c}{4 \pi \sqrt{c_{0}}} \sqrt{\sin \tilde{\Theta}\left|\frac{\partial(\tilde{T}, \tilde{\Theta}, \tilde{\Psi})}{\partial(x, y, z)}\right|}
$$

where $\tilde{T}=\tilde{T}(z ; x, y), \tilde{\Theta}=\tilde{\Theta}(z ; x, y)$ and $\tilde{\Psi}=\tilde{\Psi}(z ; x, y)$ are the arrival-time, take-off angles of $\theta$ and $\psi$ from the point source located at $\mathbf{x}=\mathbf{x}_{s}$, respectively; see Appendix for derivation.

Following the approach in [29], we first extend all the above functions into phase space, denoted as $T, \Theta$ and $\Psi$, respectively. Using (3.2) and (3.4), we obtain

$$
A(z ; x, y, \theta, \psi)=\frac{1}{4 \pi} \sqrt{\frac{c \sin \Theta}{c_{0} \sin \theta \cos \psi}} \sqrt{\frac{\Delta_{1}}{\Delta_{2}}},
$$

where $\Theta$ and $\theta$ are the takeoff angle and the arrival angle respectively, and $\Delta_{1}$ and $\Delta_{2}$ are the Jacobians of the transformation given by

$$
\Delta_{1}=\left|\begin{array}{cccc}
\phi_{x}^{1} & \phi_{x}^{2} & \Theta_{x} & \Psi_{x} \\
\phi_{y}^{1} & \phi_{y}^{2} & \Theta_{y} & \Psi_{y} \\
\phi_{\theta}^{1} & \phi_{\theta}^{2} & \Theta_{\theta} & \Psi_{\theta} \\
\phi_{\psi}^{1} & \phi_{\psi}^{2} & \Theta_{\psi} & \Psi_{\psi}
\end{array}\right|
$$

and

$$
\Delta_{2}=\left|\begin{array}{cc}
\phi_{\theta}^{1} & \phi_{\theta}^{2} \\
\phi_{\psi}^{1} & \phi_{\psi}^{2}
\end{array}\right| .
$$

See the Appendix for derivation of these formulae.

\section{Numerical Method}

4.1. Level Set Equations. One way to solve equations (3.2) and (3.4) is to use, for example, RK3 in the $z$-direction and WENO5 upwind scheme in the $x-y-\theta-\psi$ space $[22,33]$. This is a typical Eulerian approach, where grid points are fixed in space. Computational complexity of such methods is therefore $O\left(N^{5} \log N\right)$. This approach is not efficient in high dimensional space.

One reason is that, if we want to compute the multivalued arrivals from multiple point sources, we probably do not want to use some localized level set methods, like the one proposed in [27]. In this case, tracking of multiple layers of level set functions with re-initializations and extensions will take a large portion of computational time. 


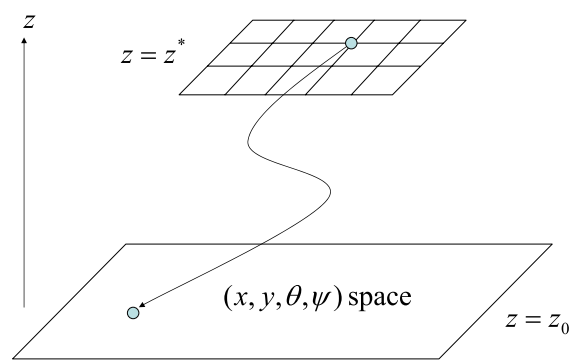

FIG. 4.1. Semi-Lagrangian method to solve the advection equations.

Another potential difficulty is the limitation from the CFL condition when solving these hyperbolic equations. For this Eulerian approach, each $z$-direction marching is of $O(\min (\Delta x, \Delta y, \Delta \theta, \Delta \psi))$. This is acceptable for lower dimensional computations, like those in [30, 29], where the dimension involved is only $1+2$ (time-like direction plus space directions). However, for the current problem, it is unreasonable to spend days of computation in solving these linear advection equations.

Therefore, we implement a semi-Lagrangian method $[36,10]$ to determine the values of $\phi^{m}$ at $z=z^{*}$ for $m=1,2$. Solving the level set equations with the method of characteristics, we have

$$
\phi^{m}=\text { const }
$$

for $m=1,2$ along the characteristics given by (2.15). Following the idea of semiLagrangian methods, we trace back in the $z$-direction until $z=z_{0}$, i.e. we solve

$$
\frac{d(\hat{x}, \hat{y}, \hat{\theta}, \hat{\psi})}{d z}=\mathbf{u}
$$

for $\left.(\hat{x}, \hat{y}, \hat{\theta}, \hat{\psi})\right|_{z=z_{0}}$ with "initial" conditions $\left.(\hat{x}, \hat{y}, \hat{\theta}, \hat{\psi})\right|_{z=z^{*}}=(x, y, \theta, \psi)$ at the current point, as shown in Figure 4.1. Then the level set values are assigned as

$$
\begin{aligned}
& \phi^{1}\left(z^{*} ; x, y, \theta, \psi\right)=\left.\hat{x}\right|_{z=z_{0}}, \\
& \phi^{2}\left(z^{*} ; x, y, \theta, \psi\right)=\left.\hat{y}\right|_{z=z_{0}},
\end{aligned}
$$

according to the initial conditions (3.3).

Numerically, the above ODE system is solved using RK3, and the step size is independent of the number of grid points used in the computational domain. By using this semi-Lagrangian approach, the computational complexity drops to $O\left(M N^{4}\right)$, where $M$ is the number of steps in the $z$-direction and $N$ is the number of grid points in each of $x-y-\theta-\psi$ direction. Different from the finite difference Eulerian approach, the factor $M$ is independent of $N$ and is chosen mainly for the purpose of accuracy. In the current implementation, we choose $M$ large enough so that the errors from the RK3 ODE solver are negligible comparing to the errors from the linear interpolation for finding intersections of level set functions on the uniform mesh. Certainly, one 


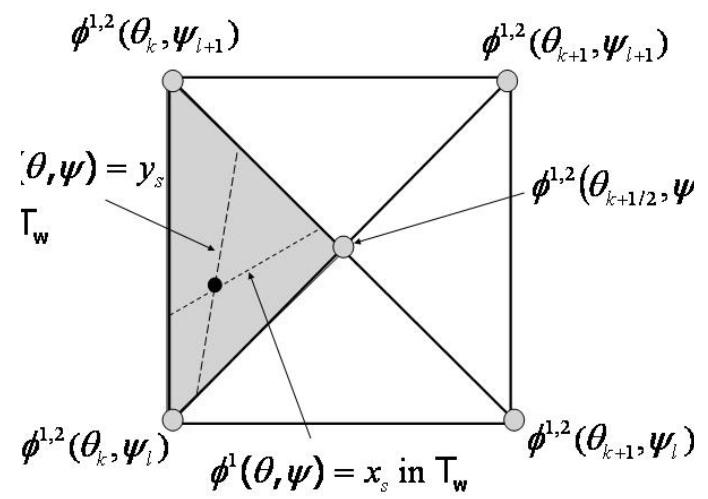

FIG. 4.2. Determining the intersection of level set functions.

may maximize the efficiency of the whole implementation by balancing the two errors from the ODE solver and the linear interpolation, but it is left for future study.

A simpler case is to consider only one point source on $z=z_{0}$. Then the order of complexity of using the global level set method approach as in [30] can still be reduced by a factor of $N^{2}$ to $O\left(N^{3} \log N\right)$ if a local level set method, for example the one from [27], is applied. However, the memory requirement, which will be addressed later in Section 4.3, may still make the local level set method difficult to implement. Moreover, to compute arrival solutions from $N^{2}$ point sources individually, one may need to solve $N^{2}$ times localized level set equations which makes the overall computational complexity back to $O\left(N^{5} \log N\right)$.

4.2. Arrival-time Equation. For the arrival-time equation, we have

$$
\frac{D T}{D z}=\frac{1}{c \sin \theta \cos \psi}
$$

where $\frac{D}{D z}$ is the material derivative given by

$$
\frac{D}{D z}=\frac{\partial}{\partial z}+\mathbf{u} \cdot \nabla=\frac{\partial}{\partial z}+x_{z} \frac{\partial}{\partial x}+y_{z} \frac{\partial}{\partial y}+\theta_{z} \frac{\partial}{\partial \theta}+\psi_{z} \frac{\partial}{\partial \psi}
$$

Therefore, we get

$$
T=\int_{\Gamma} \frac{d s}{c \sin \theta \cos \psi}
$$

where $T(0 ; x, y, \theta, \psi)=0$ by the reciprocity, and $\Gamma$ is the characteristic given by the system (4.2). Again, RK3 is used to integrate the arrival-time function of rays.

4.3. Multivalued Arrival-times. After we solve for $\phi^{m}(m=1,2)$ and $T$ on the grid points at the time level $z=z^{*}$, we need to compute the intersection of the level surface $\left\{\phi^{1}=x_{s}\right\} \cap\left\{\phi^{2}=y_{s}\right\}$. To simplify this computation, we discretize the $\theta-\psi$ space for each point $\left(x_{i}, y_{j}\right)$ in the following way. We first use rectangular grids in 
the $\theta-\psi$ space, giving $\left(\theta_{k}, \psi_{l}\right)$. One more grid point, denoted by $\left(\theta_{k+1 / 2}, \psi_{l+1 / 2}\right)$, will then be added to the center of each cell, as seen in Figure 4.2. Therefore, each grid cell with vertices at $\left(\theta_{k}, \psi_{l}\right),\left(\theta_{k+1}, \psi_{l}\right),\left(\theta_{k}, \psi_{l+1}\right)$ and $\left(\theta_{k+1}, \psi_{l+1}\right)$ will be sub-divided into 4 triangles, denoted by $\mathcal{T}_{N}, \mathcal{T}_{E}, \mathcal{T}_{S}$ and $\mathcal{T}_{W}$. For each point $\left(x_{i}, y_{j}\right)$ fixed, $\phi^{m}(m=$ $1,2)$ and $T$ will be computed at all points $\left(x_{i}, y_{j}, \theta_{k}, \psi_{l}\right)$ and $\left(x_{i}, y_{j}, \theta_{k+1 / 2}, \psi_{l+1 / 2}\right)$. On each triangle $\mathcal{T}_{(.)}, \phi^{1}$ and $\phi^{2}$ are interpolated linearly. Intersection of the level curves of $\phi^{1}$ and $\phi^{2}$ in each triangle $\mathcal{T}_{(.)},\left.\left.\left\{\phi^{1}=x_{s}\right\}\right|_{\mathcal{T}_{(.)}} \cap\left\{\phi^{2}=y_{s}\right\}\right|_{\left.\mathcal{T}_{(.)}\right)}$, is computed, if any, for every given source location $\left(x_{s}, y_{s}\right)$. The arrival-time at this intersection point will be interpolated linearly using the values of $T$ at the vertices of the triangle $\mathcal{T}_{(.)}$.

We emphasize that we need to solve each of the level set equations (3.2) and the arrival-time equation (3.4) only once, even if we care about more than one point source on $z=z_{0}$. It is the intersection of the level surfaces that we need to repeat for each of the point sources.

Another advantage of using this semi-Lagrangian method concerns the memory requirement. In the original level set method, where the level set equations are usually solved using WENO5-LF method, values at grid points are coupled together through the ENO-type reconstruction $[30,29]$. Therefore, the memory requirement would be $O\left(N^{4}\right)$.

In the Lagrangian or the semi-Lagrangian approach, on the other hand, grid points in the $x-y$ space are independent of each other throughout all processes above. Level set function values at any two points $\left(x_{i}, y_{j}, \cdot, \cdot\right)$ and $\left(x_{i^{\prime}}, y_{j^{\prime}}, \cdot, \cdot\right)$, with $i \neq i^{\prime}$ or $j \neq j^{\prime}$, are determined independently through solving a system of ODE's with different initial conditions. Although points in the $\theta-\psi$ space are dependent upon each other through the processes in determining $\Sigma_{\mathbf{x}_{s}}$ and $T\left(z^{*} ; \Sigma_{\mathbf{x}_{s}}\right)$, numerically the memory allocation can still be reduced to $O\left(N^{2}\right)$ and the computational complexity is kept to be $O\left(M N^{4}\right)$, as long as the locations of $O\left(N^{2}\right)$ multiple point sources are given at the beginning.

This reduction in the memory requirement may not be significant in the formulation as in $[30,29]$. However, it is important in the current implementation in the $1+4$ dimensions (time-like direction plus $x-y-\theta-\psi$ directions). Parallel computation is also possible, although it is not implemented at this moment.

4.4. Reinitialization and Intersection. In our current formulation, all the values of the level set functions are used rather than only the zero value. The usual reinitialization is a process of reconstructing a signed distance function to the zero level set so that the only useful information from the original level set function is concentrated at/near the zero level set. Therefore, the information at other places is no longer meaningful. Hence, it is impossible to apply such a technique in our setup.

On the other hand, we have to find the intersections of the level sets eventually, i.e. to determine (3.7). Since we use a semi-Lagrangian method to solve the level set equations, the accuracy of the level set functions solely depends on the ODE solver rather than on the number of grid points in the $(x, y, \theta, \psi)$ space. However, to obtain accurate intersections of both zero level sets and non-zero level sets, we may refine the grids in the $(\theta, \psi)$ space, as illustrated in Figure 4.2 .

4.5. Amplitude. We notice that the takeoff angles $\Theta$ and $\Psi$ are the byproducts in the above computations of $\phi^{1}$ and $\phi^{2}$. We can simply set

$$
\begin{aligned}
& \Theta\left(z^{*} ; x, y, \theta, \psi\right)=\hat{\theta}\left(z=z_{0}\right) \\
& \Psi\left(z^{*} ; x, y, \theta, \psi\right)=\hat{\psi}\left(z=z_{0}\right) .
\end{aligned}
$$


However, to compute $\Delta_{1}$, instead of numerically differentiating these four functions with respect to $x, y, \theta$ and $\psi$ respectively, we notice that this quantity satisfies the equation

$$
\frac{D \Delta_{1}}{D z}=-(\nabla \cdot \mathbf{u}) \Delta_{1}
$$

where $\Delta_{1}(0 ; x, y, \theta, \psi)=1$ by the reciprocity principle and

$$
\nabla \cdot \mathbf{u}=\frac{\left(1+\cos ^{2} \psi\right) c_{z}+\left(\tan \psi \cos ^{2} \psi\right) c_{y}}{c \sin ^{2} \theta \cos ^{2} \psi} .
$$

In turn, we solve this equation by introducing $\tilde{\Delta}_{1}=\log \left(\Delta_{1}\right)$. Then, similar to the computation of the arrival-time, we integrate

$$
\tilde{\Delta}_{1}=\int_{\Gamma}-(\nabla \cdot \mathbf{u}) d s
$$

with $\tilde{\Delta}_{1}(0 ; x, y, \theta, \psi)=0$ using RK3. Finally, we have

$$
\Delta_{1}=\exp \left(\tilde{\Delta}_{1}\right) .
$$

For the quantity $\Delta_{2}$, we can simply use the linear interpolants of $\phi^{1}$ and $\phi^{2}$ in the triangle $\mathcal{T}_{(\text {.) }}$ when determining $\Sigma_{\mathbf{x}_{s}}$. Assuming that those linear interpolants are

$$
\begin{aligned}
& \phi_{\mathcal{T}}^{1}=a_{11} \theta+a_{12} \psi+b_{1}, \\
& \phi_{\mathcal{T}}^{2}=a_{21} \theta+a_{22} \psi+b_{2},
\end{aligned}
$$

we have $\Delta_{2}=\left|a_{11} a_{22}-a_{12} a_{21}\right|$ defined on the triangle $\mathcal{T}_{(.)}$.

To compute $A\left(z^{*} ; \Sigma_{\mathbf{x}_{s}}\right)$, we first determine the quantity

$$
\alpha=\frac{\Delta_{1} \sin \Theta}{\cos \psi \sin \theta}
$$

at each grid point. Next we use linear interpolation to find its value at the intersection $\left.\left.\left\{\phi^{1}=x_{s}\right\}\right|_{\mathcal{T}_{(.)}} \cap\left\{\phi^{2}=y_{s}\right\}\right|_{\mathcal{T}_{(\cdot)}}$.

Overall, we have

$$
A\left(z^{*} ; \Sigma_{\mathbf{x}_{s}}\right)=\frac{1}{4 \pi} \sqrt{\frac{c}{c_{s}}} \sqrt{\frac{(\alpha)_{\Sigma_{\mathbf{x}_{s}}}}{\left(\Delta_{2}\right)_{\mathcal{T}_{(.)}}}} .
$$

\section{Numerical Examples}

In the following numerical examples, the computational domain is chosen to be

$$
\Omega=\{(x, y, \theta, \psi): x \in[-1,1], y \in[-1,1], \theta \in[\pi / 20,19 \pi / 20], \psi \in[-9 \pi / 20,9 \pi / 20]\} .
$$

Multiple point sources are located on $z=0$. Their coordinates $\left(x_{s}, y_{s}\right)(s=1, \cdots$, $4)$ are given by $(0,0),(0.3,0.4),(-0.1,0.2)$ and $(0.2,-0.3)$. 

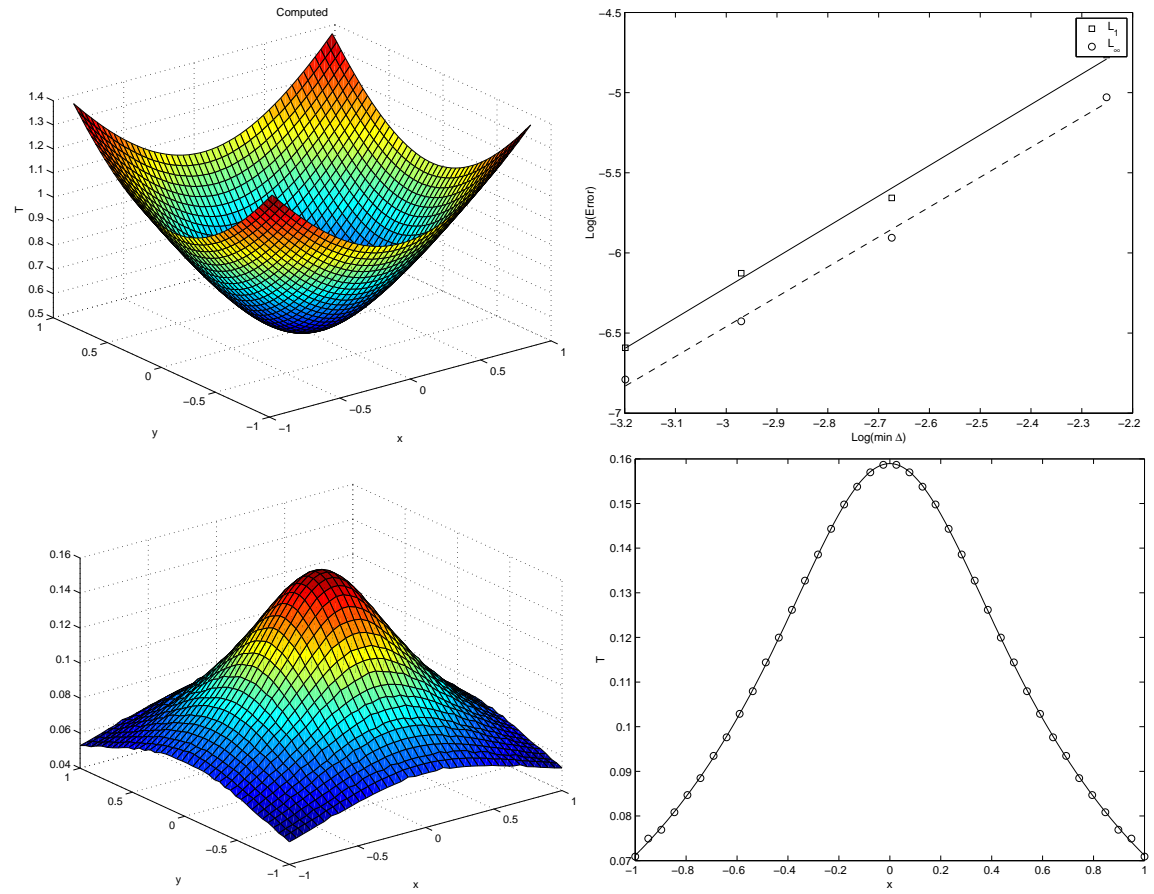

FIG. 5.1. (Example 1) Computed arrival-time in physical space using $1 \leq i, j, k, l \leq 50$ grids in phase space at $z=0.5$ and the corresponding convergence test result. Second row shows the amplitude of the arrival rays at the same $z$ in physical space using $1 \leq i, j \leq 40$ and $1 \leq k, l \leq 200$. Last sub-figure shows the amplitude at the cross section, $y=0$, together with the exact solution.

5.1. Example 1: Constant Velocity Model. This example is a constant velocity model where the velocity field is given by $c \equiv 1$. Exact solution of arrival-times is given by

$$
T(z ; x, y)=\sqrt{z^{2}+x^{2}+y^{2}},
$$

which is the distance function to the point source at the origin.

The sub-figures on the first row of Figure 5.1 show the computed solution. Convergence rate of the proposed method is also shown on the same row. We performed computations using $M=5$ and $N=10 n$ for $n=2$ to 5 , where $M$ is the number of steps in the ODE solver and $N$ is the number of mesh points in every one of the $x, y, \theta$ and $\psi$ directions. The $x$-axis on the graph represents the logarithm value of $\Delta x \Delta y$, and the $y$-axis is the logarithm value of the error of the solution measured in the $L_{1}$-norm and the $L_{\infty}$-norm. The numerical rates of convergence, twice of the slopes of the least-squares fitted lines, are 1.90 and 1.86 in terms of two different norms.

The sub-figures in the second row show some results for the amplitude. To have accurate results, we use 4 times the number of grids in the $\theta-\psi$ space as that in the $x-y$ space. We also plot the computed solution (in circles) together with the exact solution (in solid line), given by

$$
A(z ; x, y)=\frac{1}{4 \pi R}
$$

where $R$ is the distance from the point source at the origin. 

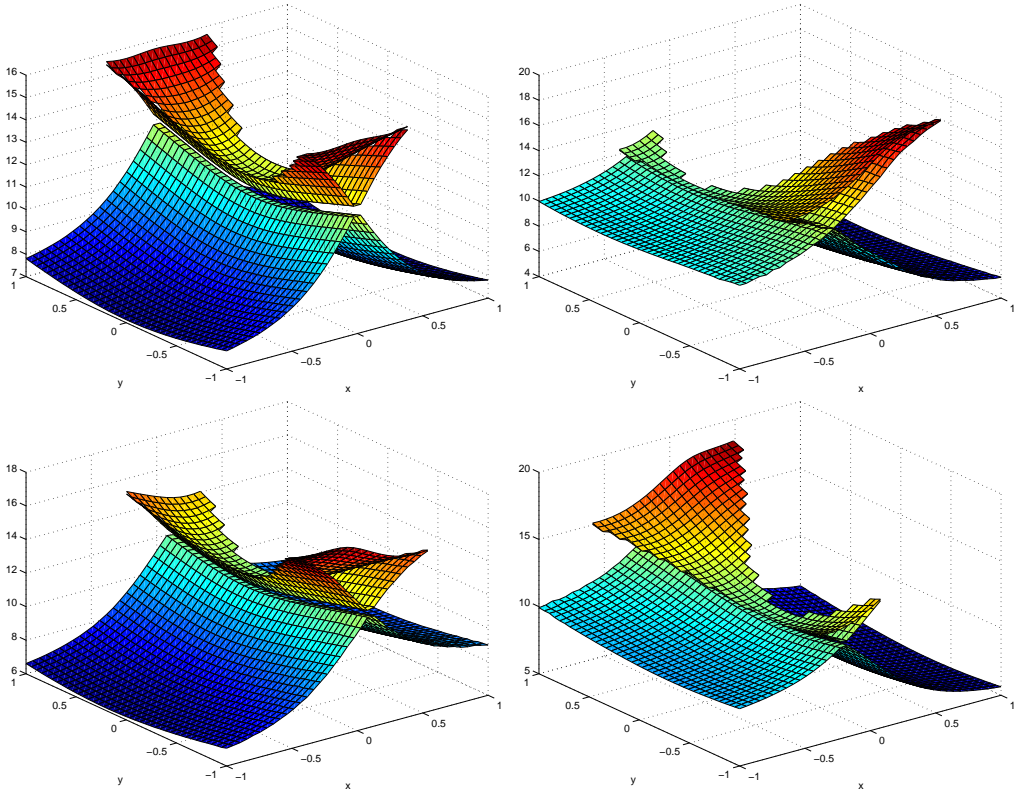

FIG. 5.2. (Example 2) Arrival-times in physical space at $z=1.2$ using $M=24,1 \leq i, j \leq 41$ and $1 \leq k, l \leq 201$ in phase space; point sources located at $\left(x_{s}, y_{s}\right)$ for $s=1, \cdots, 4$, respectively. Compare to Figure 5.3.

5.2. Example 2: Waveguide Model. This velocity model is the same as the one in $[30,29]$, where

$$
c(x, y, z)=1.1-\exp \left(-0.5 x^{2}\right) .
$$

Figure 5.2 shows the computed multivalued arrival-times at $z=1.2$ using $M=24$, $1 \leq i, j \leq 41$ and $1 \leq k, l \leq 201$ for point sources located at $\left(x_{s}, y_{s}\right)(s=1, \cdots, 4)$, respectively. As the location of point sources varies, the travel-time varies for a ray to reach a specific location. There are three sheets of travel-time surfaces at $z=1.2$ for those given point sources. When two sheets connect to each other in the phase space, they connect along the caustic curves, which are shown in the sub-figures; this can also be seen more clearly in Figure 5.4. In all the sub-figures, we can see that the caustics develop and the travel-time becomes triple valued around caustics.

Solutions using the ray tracing method are given in Figure 5.3. Rays are emitted from the point sources located at $\left(x_{s}, y_{s}\right)$ with initial take-off angles $(\theta, \psi)$ uniformly sampled in the angle space with a 50 by 50 grid. The paraxial ray tracing system (2.15) is then solved using RK45 until $z=1.2$. As expected, we can not have uniform resolution of the solution. Rays are more concentrated on the later arrivals. Solutions from the first arrivals, on the other hand, are resolved relatively poorly. Comparing Figure 5.2 and Figure 5.3, the solutions have the same structure.

Because of the non-uniform resolution in the ray-tracing solution, it is difficult to compare the solutions from the current level set formulation with that from the ray tracing method directly. However, since in this case $c_{y}=c_{z}=0$, this implies

$$
u^{3}=\frac{c_{x}}{c \cos \psi} \text { and } u^{4}=0 .
$$



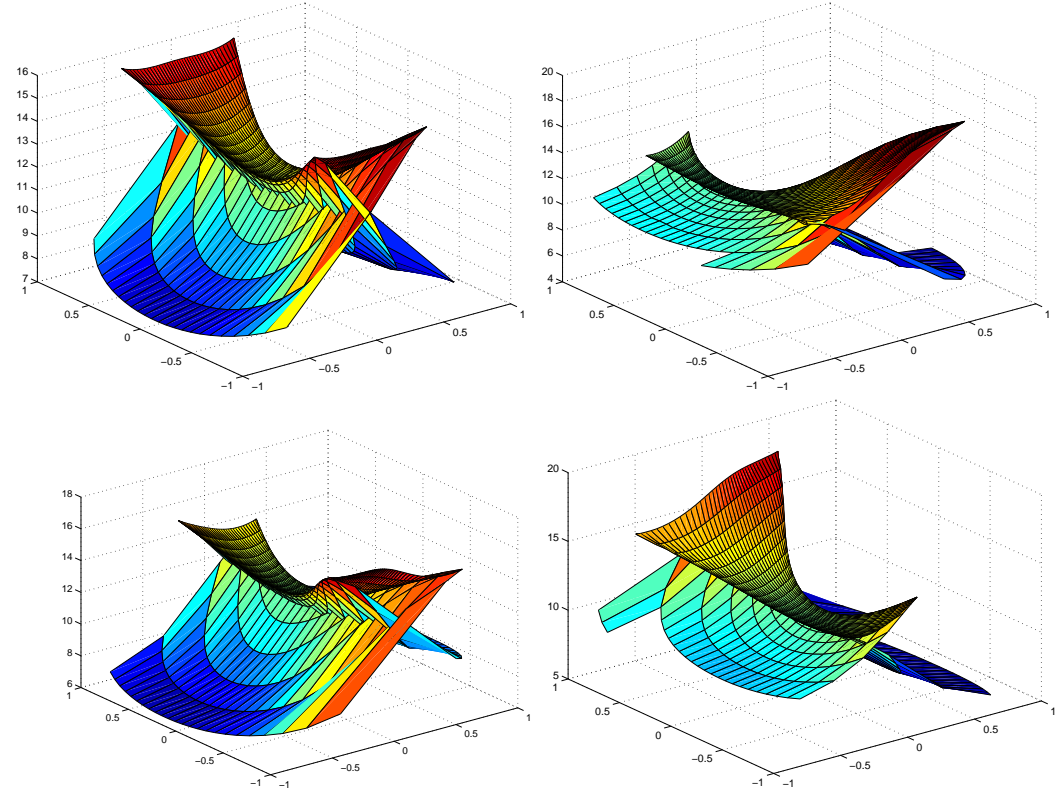

FIG. 5.3. (Example 2) Arrival-times in physical space at $z=1.2$ using ray tracing method; point sources located at $\left(x_{s}, y_{s}\right)$ for $s=1, \cdots, 4$, respectively. Compare to Figure 5.2.
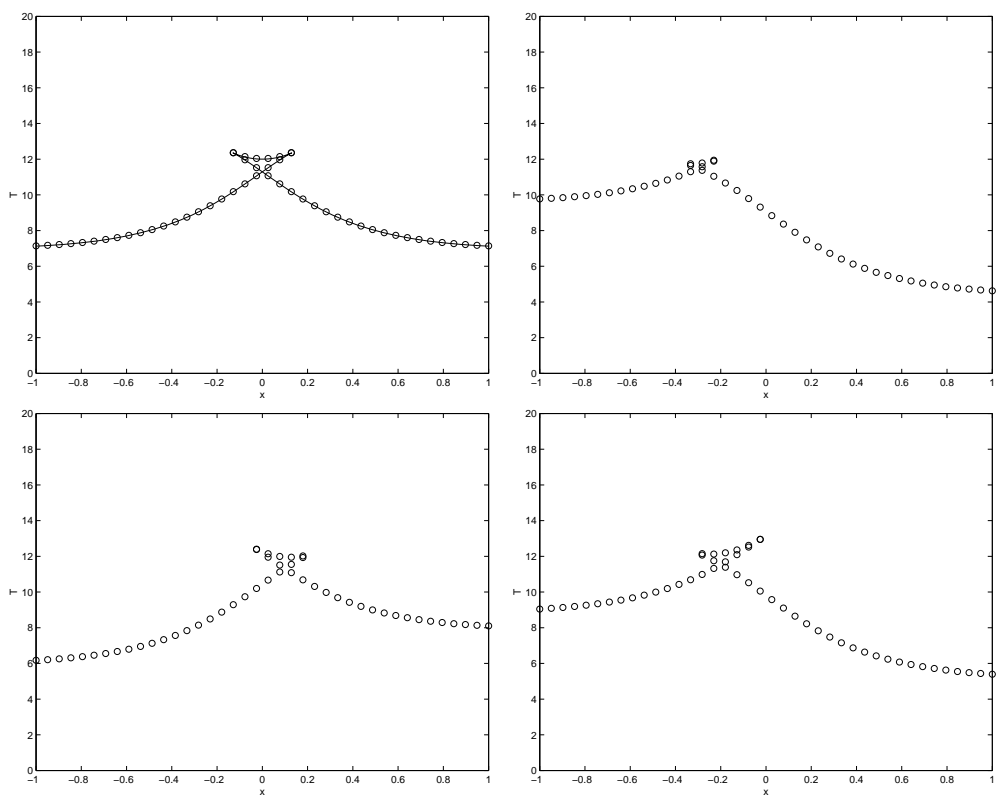

Fig. 5.4. (Example 2) Arrival-times in physical space at $z=1.2$ on the cross section $y=0$ using $M=24,1 \leq i, j \leq 41$ and $1 \leq k, l \leq 201$ in phase space; point sources located at $\left(x_{s}, y_{s}\right)$ for $s=1, \cdots, 4$, respectively. Solution from the ray tracing method plotted using solid line in the first sub-figure. 

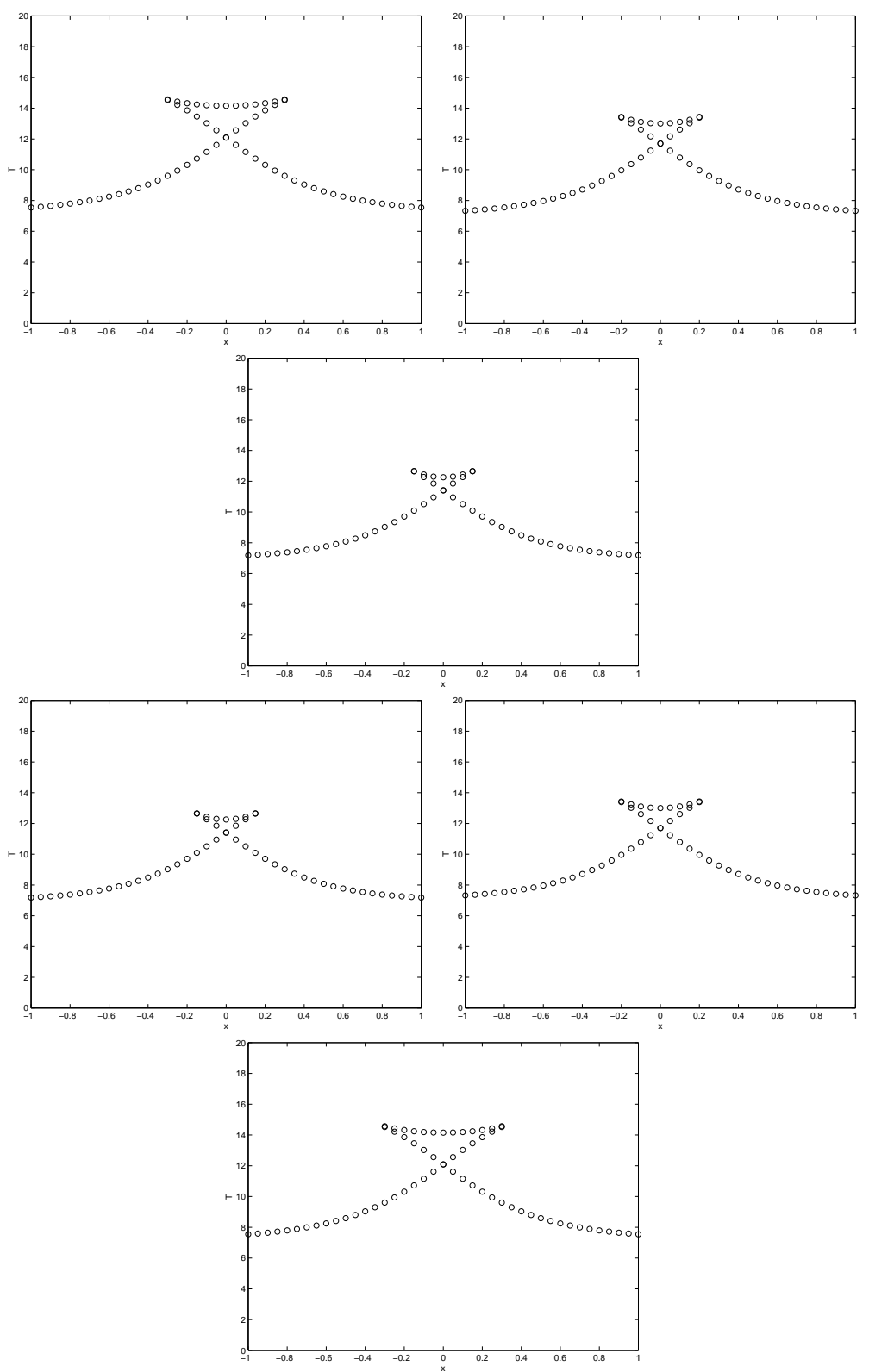

FIG. 5.5. (Example 2) Arrival-times in physical space at $z=1.2$ on different cross sections $y=(j-21) / 20$ for $j=6,11,16,26,31,36$ using $M=24,1 \leq i, j \leq 41$ and $1 \leq k, l \leq 201$ in phase space; $a$ point source located at $\left(x_{s}, y_{s}\right)=(0,0)$.

Considering rays shooting out from the origin with $\psi=0$ and using the paraxial ray tracing system $(2.15)$, we get $\psi(z) \equiv 0$. This means that all the rays with $\psi(z=$ 0 ) $=0$ from the origin will stay on the cross section $y=0$ (one can compare our solution with that in $[30,29])$. We compare the solution of this cross section with the one from the ray tracing method, as shown in the first sub-figure of Figure 5.4. The solution 

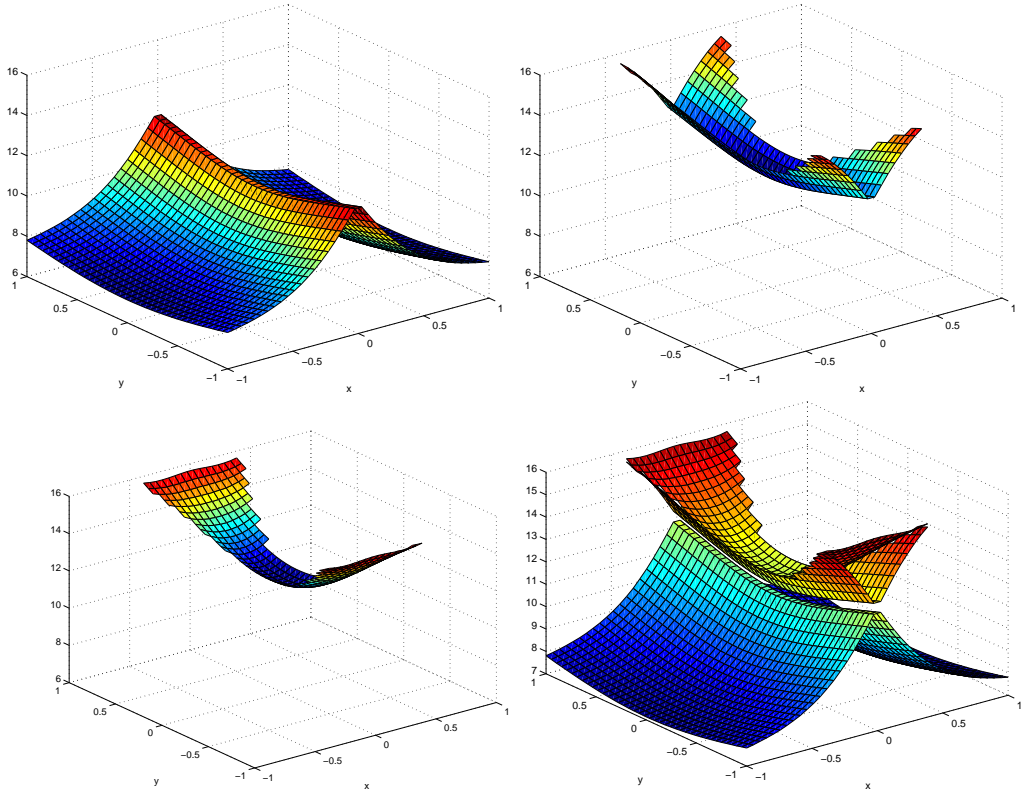

Fig. 5.6. (Example 2) First, second and third arrival-times in physical space at $z=1.2$ using $M=24,1 \leq i, j \leq 41$ and $1 \leq k, l \leq 201$ in phase space; a point source located at $\left(x_{s}, y_{s}\right)=(0,0)$.

from the ray tracing method is plotted in the solid line, while the circles represent the solution from the current formulation. They match with each other very well.

To look at solutions more closely, we concentrate on the solution for the point source located at $\left(x_{s}, y_{s}\right)=(0,0)$. More cross sections of the multivalued arrivaltime are plotted in Figure 5.5. The locations of the slices are $y=(j-21) / 20$ for $j=6,11,16,26,31,36$. One can imagine that the leftmost slice is shifted from the left to the right and scaled according to the distance to the origin, since the velocity is a function of the $x$ variable only.

Figure 5.6 shows the surface plots of the arrival-time solutions according to the arrival orders. If the eikonal equation is solved directly, one would get the viscosity solution, which corresponds to the first arrival-time, i.e. the solution on the first sub-figure.

Amplitudes of the arrival rays are also calculated. Figures 5.7 to 5.10 show the amplitude solutions corresponding to the corresponding results in Figures 5.2, 5.4 to 5.6 .

In the calculation of the amplitude, one needs to calculate the Jacobian $\Delta_{2}$. This quantity can be zero which corresponds to the location of caustics. Near caustics, the usual asymptotic expansion of the wave field is not valid anymore. This reflects in the fact that the amplitude blows up, as seen clearly in these figures.

In Figure 5.10, we can see that the amplitude becomes infinity along the caustic curves in the sub-figures of amplitudes corresponding to the second and third arrivals. 
5.3. Example 3: Vinje's Gaussian Model. This velocity model comes from [40] where

$$
c(x, y, z)=3-1.75 \exp \left(-\frac{x^{2}+y^{2}+(z-0.75)^{2}}{0.5^{2}}\right) .
$$
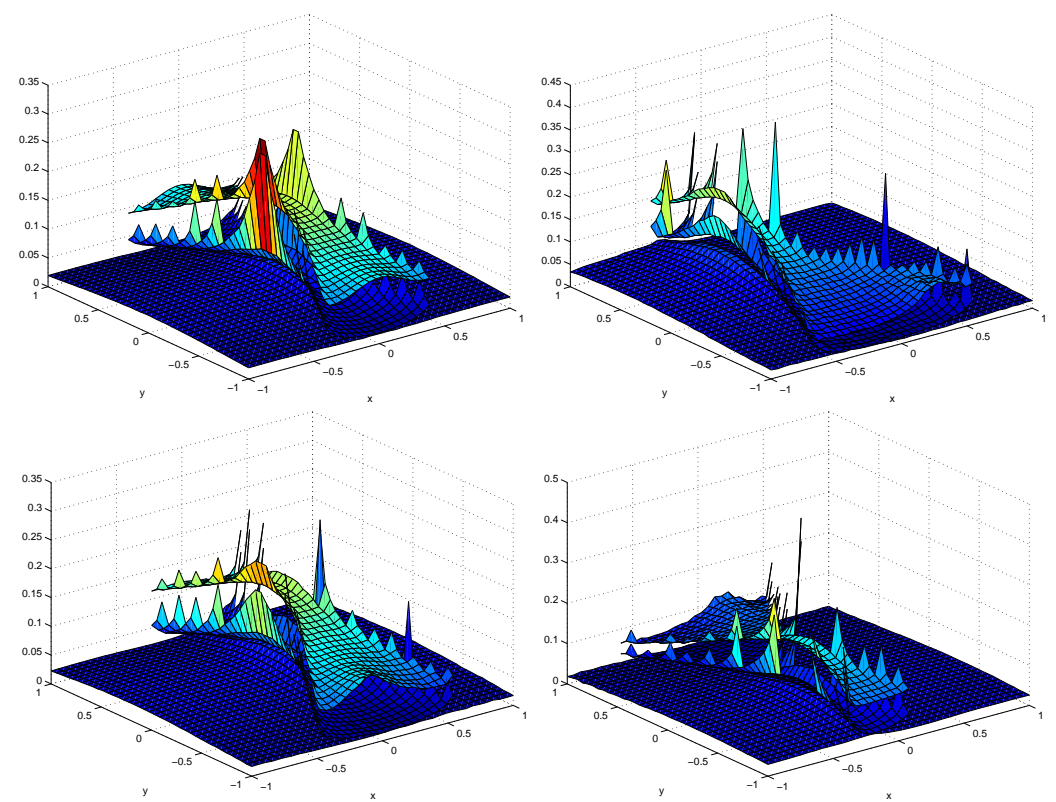

FIG. 5.7. (Example 2) Amplitudes in physical space at $z=1.2$ using $M=24,1 \leq i, j \leq 41$ and $1 \leq k, l \leq 201$ in phase space; point sources located at $\left(x_{s}, y_{s}\right)$ for $s=1, \cdots, 4$, respectively.

Figure 5.11 shows the multivalued arrival-times at $z=1.5$ using $M=15,1 \leq i, j \leq$ 51 and $1 \leq k, l \leq 401$ with sources located at $\left(x_{s}, y_{s}\right)$ for $s=1, \cdots, 4$, respectively. As the source varies, the travel-time varies for a ray to reach a specific location. There are three sheets of travel-time surfaces at $z=1.5$ for those given sources. When two sheets connect to each other in phase space, they connect along the caustic curves, which are shown in the figures; this can also be seen more clearly in Figure 5.13.

Solutions using ray tracing method are given in Figure 5.12. Rays are emitted from the point sources located at $\left(x_{s}, y_{s}\right)$ with initial take-off angles $(\theta, \psi)$ uniformly sampled in the angle space with a 50 by 50 grid. The paraxial ray tracing system (2.15) is then solved using RK45 until $z=1.5$. As expected, we can not have uniform resolution of the solution. Rays are more concentrated on the later arrivals. Solutions from the first arrivals, on the other hand, are resolved relatively poorly. Comparing Figure 5.11 and Figure 5.12, the solutions have the same structure.

In this case, although $u^{4}$ cannot be simplified to 0 , rays from the origin with the initial $\psi=0$ are still staying on the cross section $y=0$. From the last equation in (2.15), if $\psi(z=0)=0$ and $y=0$, which implies $c_{y}=0$, we can obtain $\psi(z) \equiv 0$. Therefore, we can still compare our solution with the one obtained from the ray tracing method on the cross section $y=0$, as shown in Figure 5.13. Again, the solid line represents the solution using the ray tracing method. The solutions by the method presented here 
are plotted using circles. Two solutions match with each other very well. In all the sub-figures, we can see that the caustics develops and the travel-time becomes triple valued around caustics.

To look at the solutions more closely, we concentrate on the solution for the source located at $\left(x_{s}, y_{s}\right)=(0,0)$. More cross sections of the multivalued arrival-time are plotted on Figure 5.14. The locations of the slices are $y=(j-26) / 25$ for $j=$ $11,21,31,41$. One can see the obvious symmetry since the velocity has rotational invariance for $z$ fixed.

Figure 5.15 shows the surface plots of the arrival-time solutions according to the arrival orders. If the eikonal equation is solved directly, one would get the viscosity solution, which corresponds to the first arrival-time, i.e. the solution on the first sub-figure.

Amplitudes of the arrival rays are also calculated. Figures 5.16 to 5.19 show the amplitude solutions corresponding to Figures 5.11, 5.13 to 5.15.

In Figure 5.19, we can see that the amplitude becomes infinity along the caustic lines in the sub-figures of amplitudes corresponding to the second and third arrivals.

\section{Conclusion}

We developed a level set method to compute the three dimensional multivalued geometrical optics term in the paraxial formulation. This method has two new features: it does not require reinitialization and it can handle multiple point sources simultaneously. By using a semi-Lagrangian method in the paraxial formulation, the method has $O\left(N^{2}\right)$ rather than $O\left(N^{4}\right)$ memory storage requirement for up to $O\left(N^{2}\right)$ multiple point sources in the five dimensional phase space, where $N$ is the number of mesh points along one direction. Although the computational complexity is
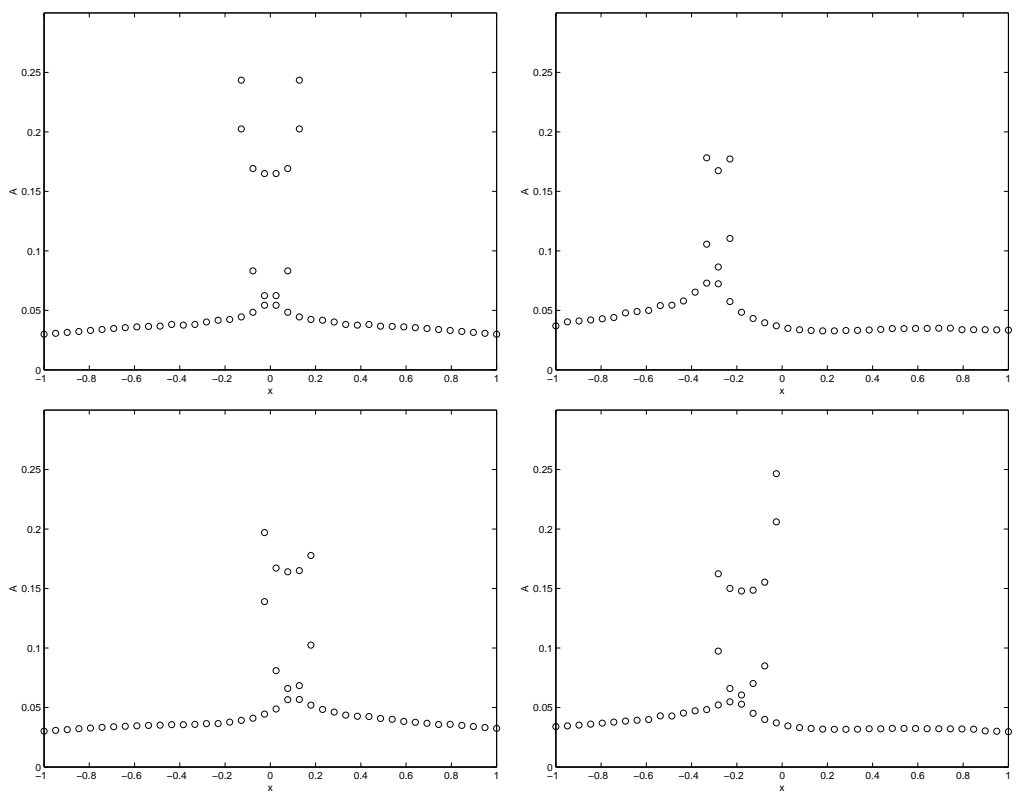

FIG. 5.8. (Example 2) Amplitudes in physical space at $z=1.2$ on the cross section $y=0$ using $M=24,1 \leq i, j \leq 41$ and $1 \leq k, l \leq 201$ in phase space; point sources located at $\left(x_{s}, y_{s}\right)$ for $s=1, \cdots, 4$, respectively. 


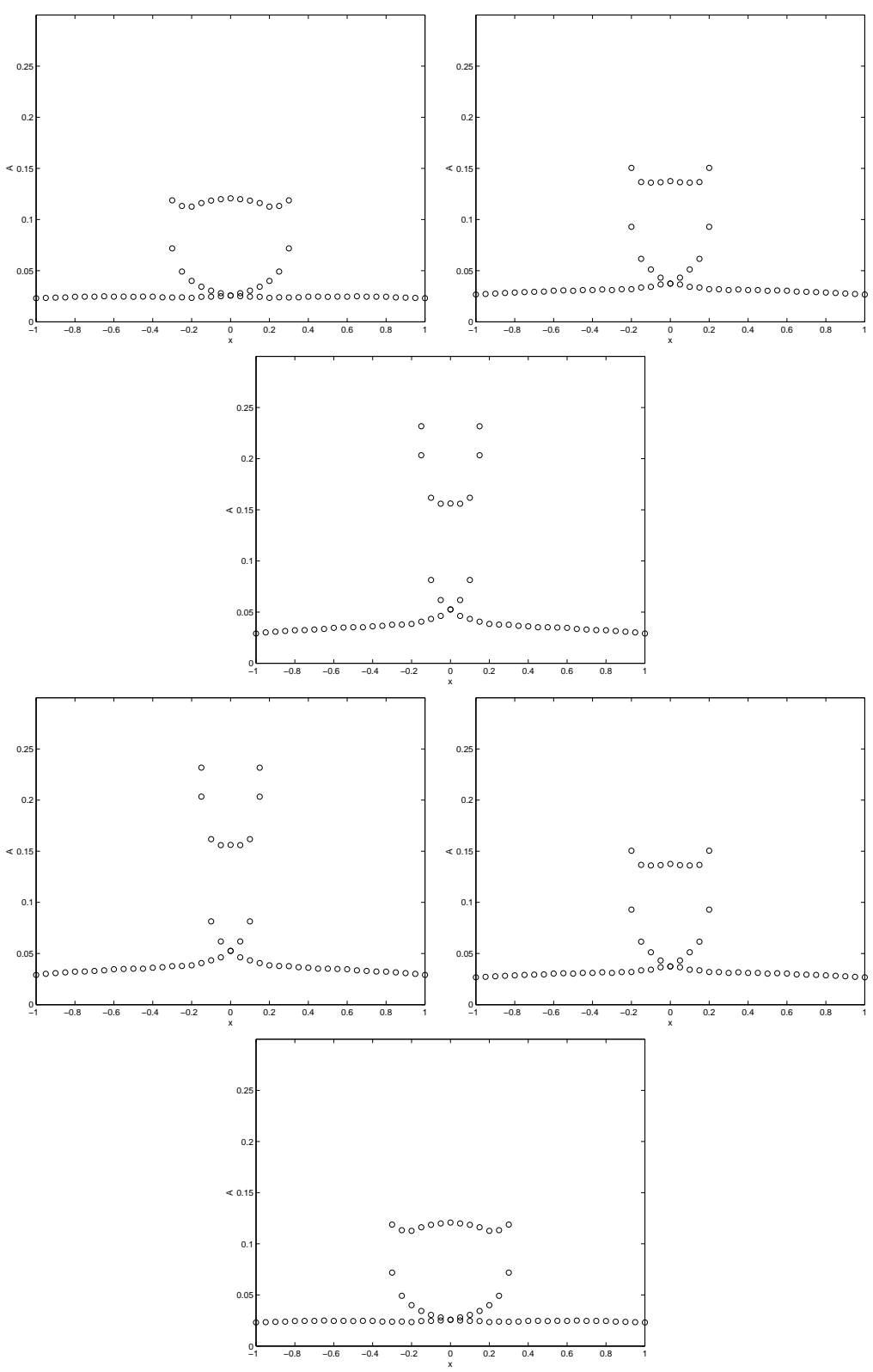

FIG. 5.9. (Example 2) Amplitudes in physical space at $z=1.2$ on different cross sections $y=$ $(j-21) / 20$ for $j=6,11,16,26,31,36$ using $M=24,1 \leq i, j \leq 41$ and $1 \leq k, l \leq 201$ in phase space; point sources located at $\left(x_{s}, y_{s}\right)$ for $s=1, \cdots, 4$, respectively.

still $O\left(M N^{4}\right)$, this disadvantage is largely overcome by the fact that $O\left(N^{2}\right)$ multiple point sources can be treated simultaneously. Numerical examples demonstrated the efficiency and accuracy of the new method. 

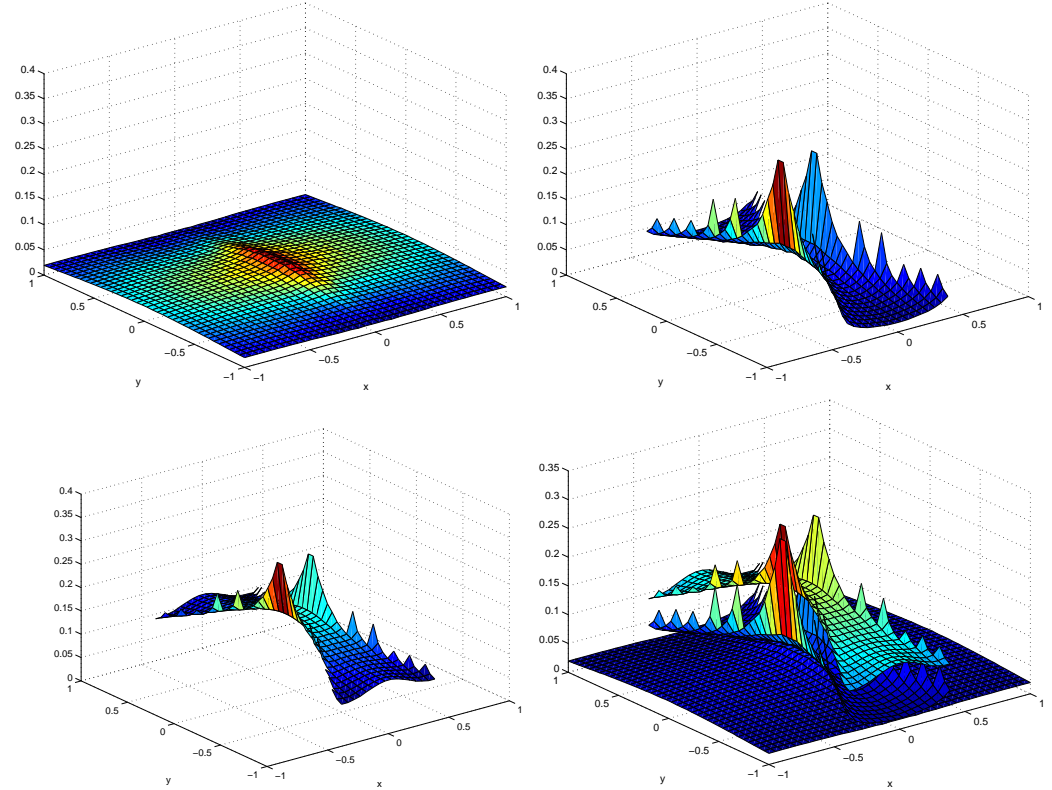

FIG. 5.10. (Example 2) First, second and third arrival amplitudes in physical space at $z=1.2$ using $M=24,1 \leq i, j \leq 41$ and $1 \leq k, l \leq 200$ in phase space; a point source located at $\left(x_{s}, y_{s}\right)=(0,0)$.
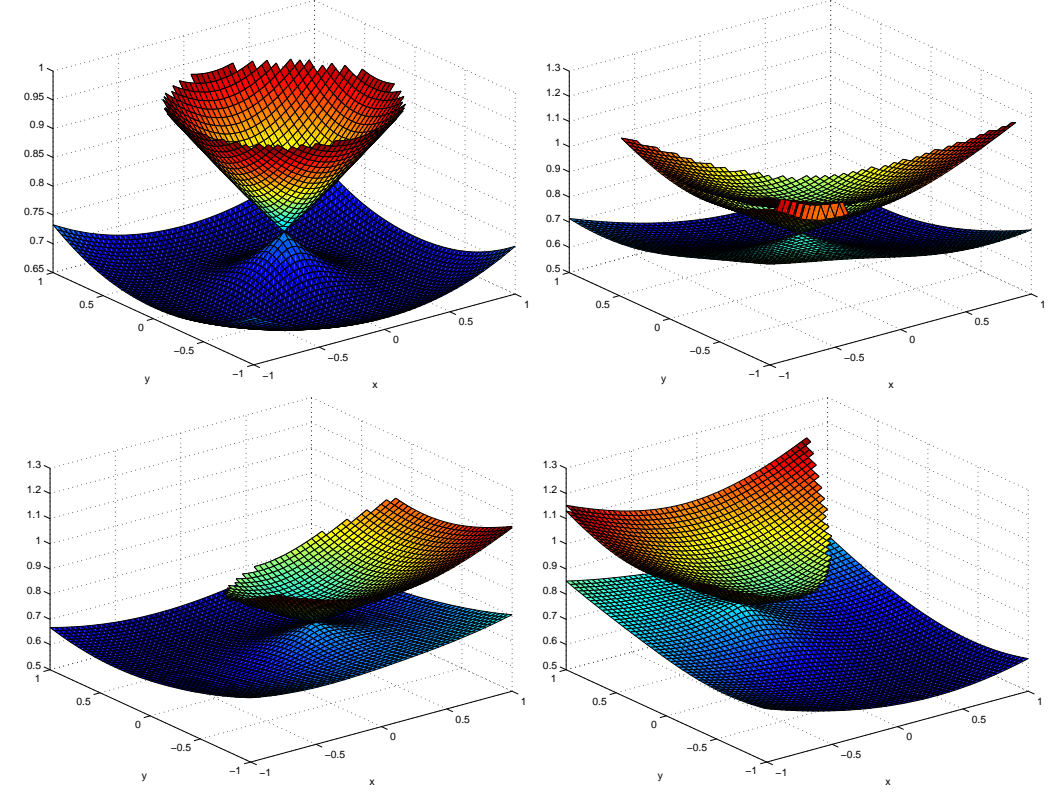

FIG. 5.11. (Example 3) Arrival-times in physical space at $z=1.5$ using $M=15,1 \leq i, j \leq 51$ and $1 \leq k, l \leq 401$ in phase space; point sources located at $\left(x_{s}, y_{s}\right)$ for $s=1, \cdots, 4$, respectively. Compare to Figure 5.12. 

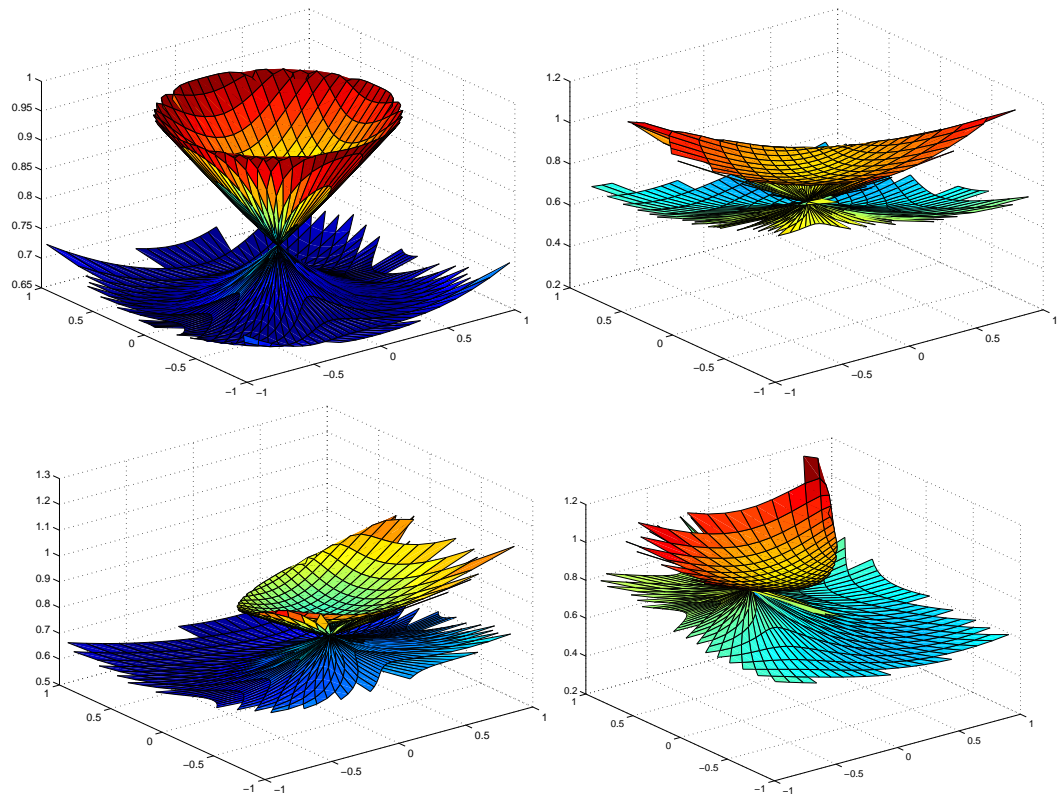

Fig. 5.12. (Example 3) Arrival-times in physical space at $z=1.5$ using a ray tracing method; point sources located at $\left(x_{s}, y_{s}\right)$ for $s=1, \cdots, 4$, respectively. Compare to Figure 5.11.
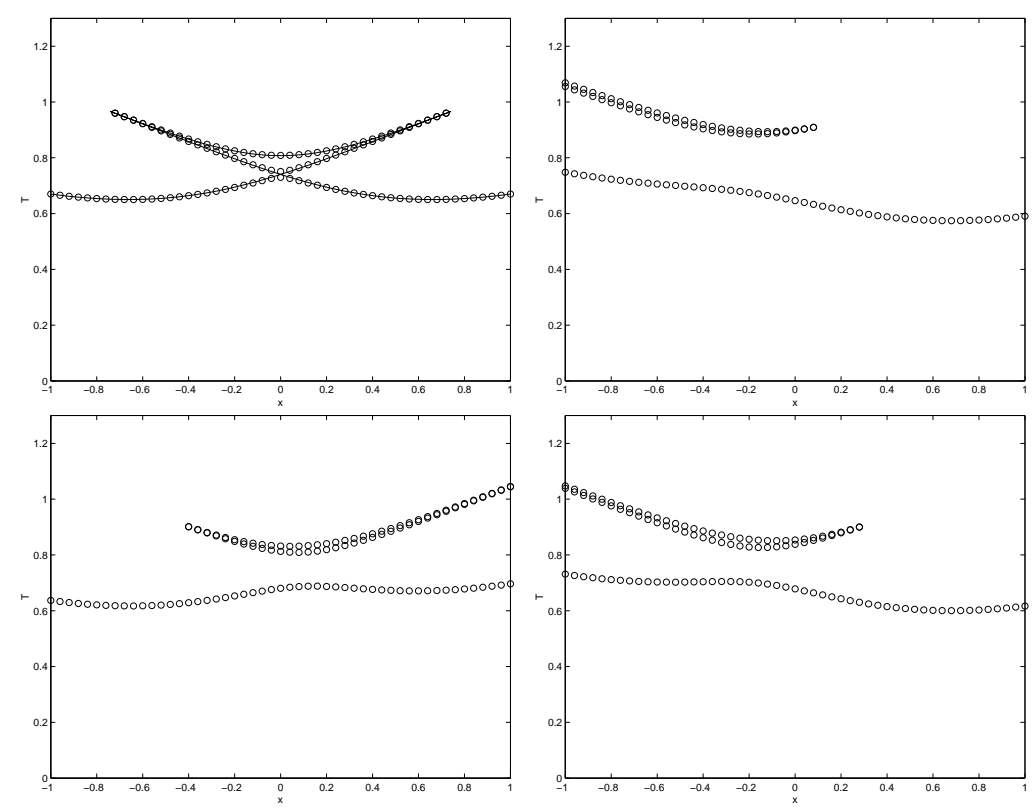

FIG. 5.13. (Example 3) Arrival-times in physical space at $z=1.5$ on the cross section $y=0$ using $M=15,1 \leq i, j \leq 51$ and $1 \leq k, l \leq 401$ in phase space; point sources located at $\left(x_{s}, y_{s}\right)$ for $s=1, \cdots, 4$, respectively. Solution using ray tracing method plotted in solid line in the first sub-figure. 

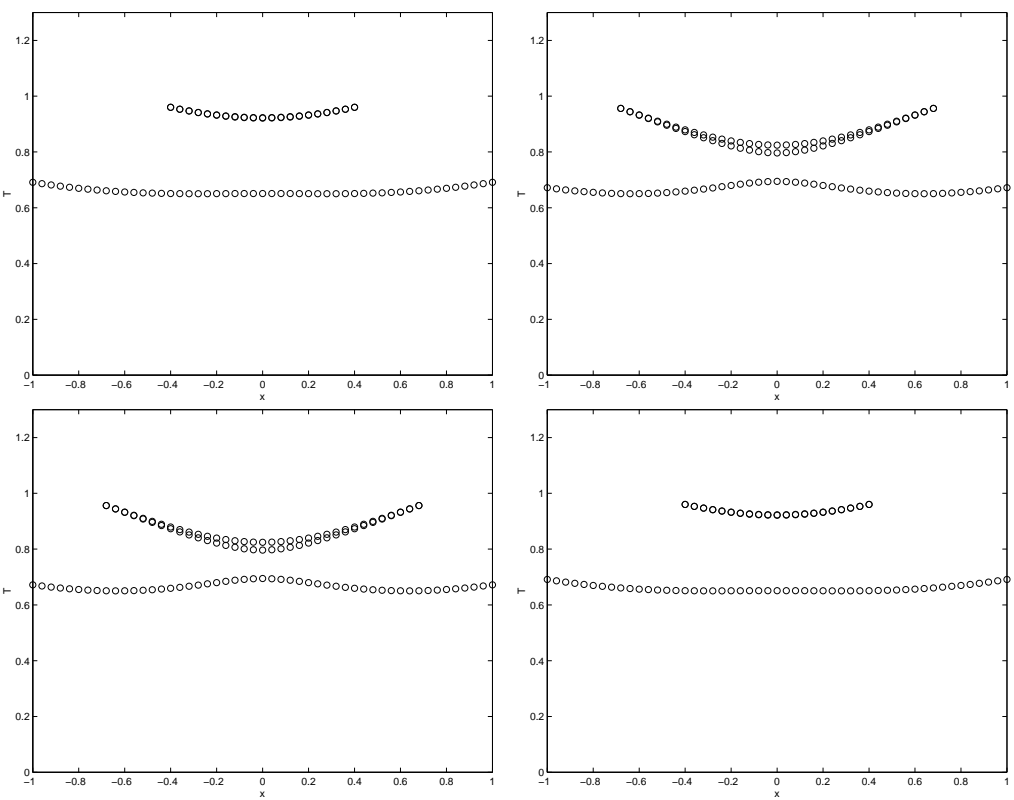

Fig. 5.14. (Example 3) Arrival-times in physical space at $z=1.5$ on different cross sections $y=(j-26) / 25$ for $j=11,21,31,41$ using $M=15,1 \leq i, j \leq 51$ and $1 \leq k, l \leq 401$ in phase space; a point source located at $\left(x_{s}, y_{s}\right)=(0,0)$.
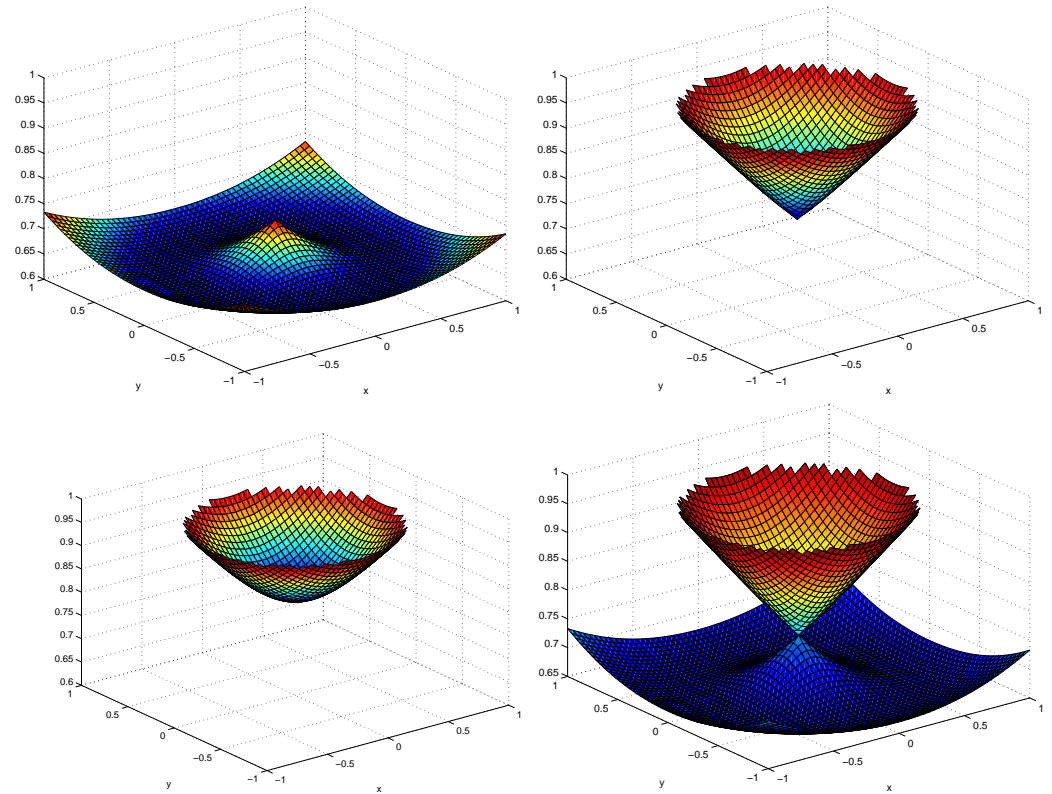

FIG. 5.15. (Example 3) First, second and third arrival-times in physical space at $z=1.5$ using $M=15,1 \leq i, j \leq 51$ and $1 \leq k, l \leq 401$ in phase space; a point source located at $\left(x_{s}, y_{s}\right)=(0,0)$. 

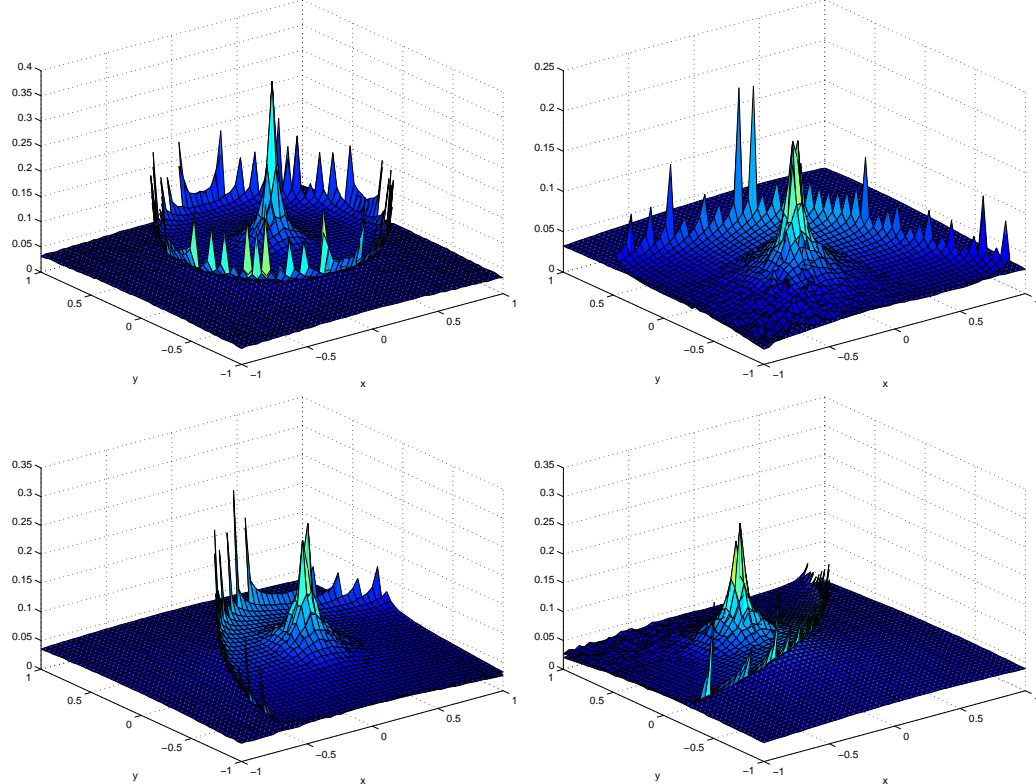

FIG. 5.16. (Example 3) Amplitudes in physical space at $z=1.5$ using $M=15,1 \leq i, j \leq 51$ and $1 \leq k, l \leq 401$ in phase space; point sources located at $\left(x_{s}, y_{s}\right)$ for $s=1, \cdots, 4$, respectively.
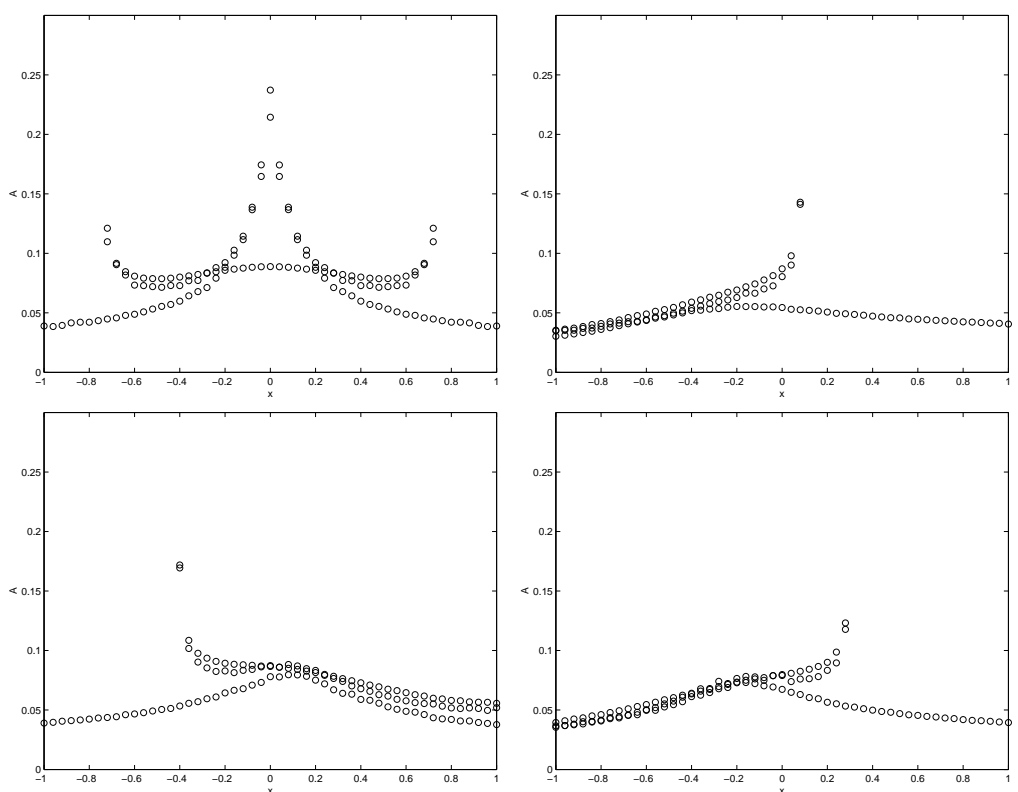

FIG. 5.17. (Example 3) Amplitudes in physical space at $z=1.5$ on the cross section $y=0$ using $M=15,1 \leq i, j \leq 51$ and $1 \leq k, l \leq 401$ in phase space; point sources located at $\left(x_{s}, y_{s}\right)$ for $s=1, \cdots, 4$, respectively. 
668

A LEVEL SET METHOD FOR PARAXIAL GEOMETRICAL OPTICS
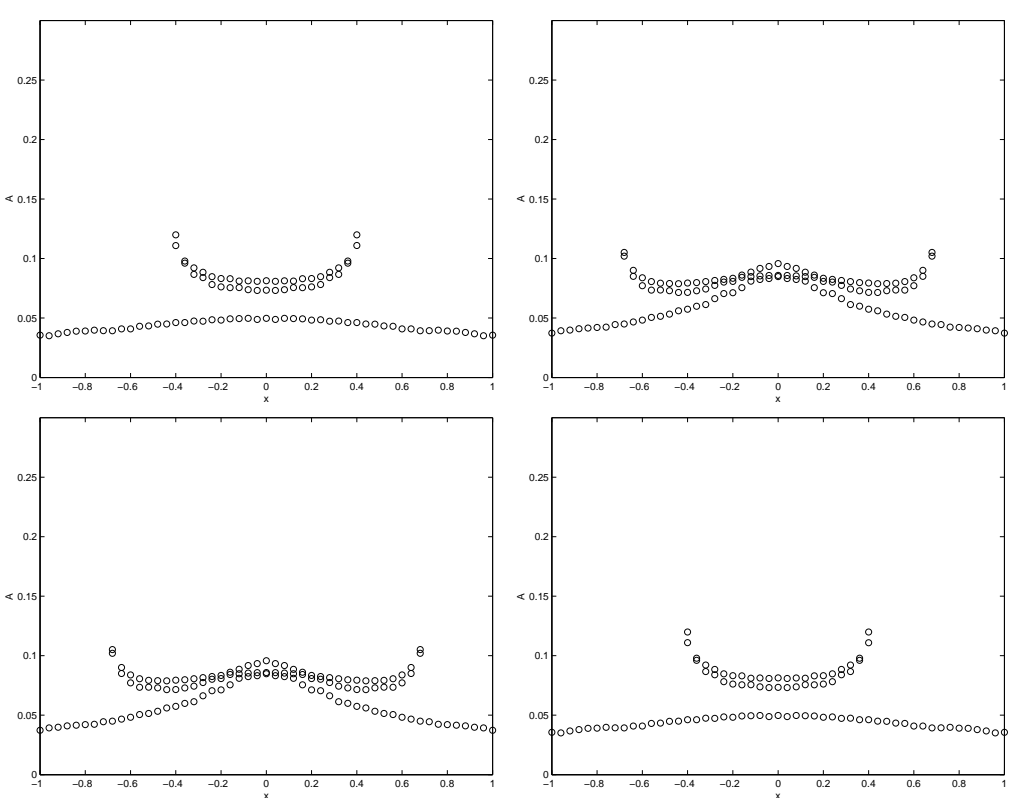

FIG. 5.18. (Example 3) Amplitudes in physical space at $z=1.5$ on different cross sections $y=(j-26) / 25$ for $j=11,21,31,41$ using $M=15,1 \leq i, j \leq 51$ and $1 \leq k, l \leq 401$ in phase space; a point source located at $\left(x_{s}, y_{s}\right)=(0,0)$.
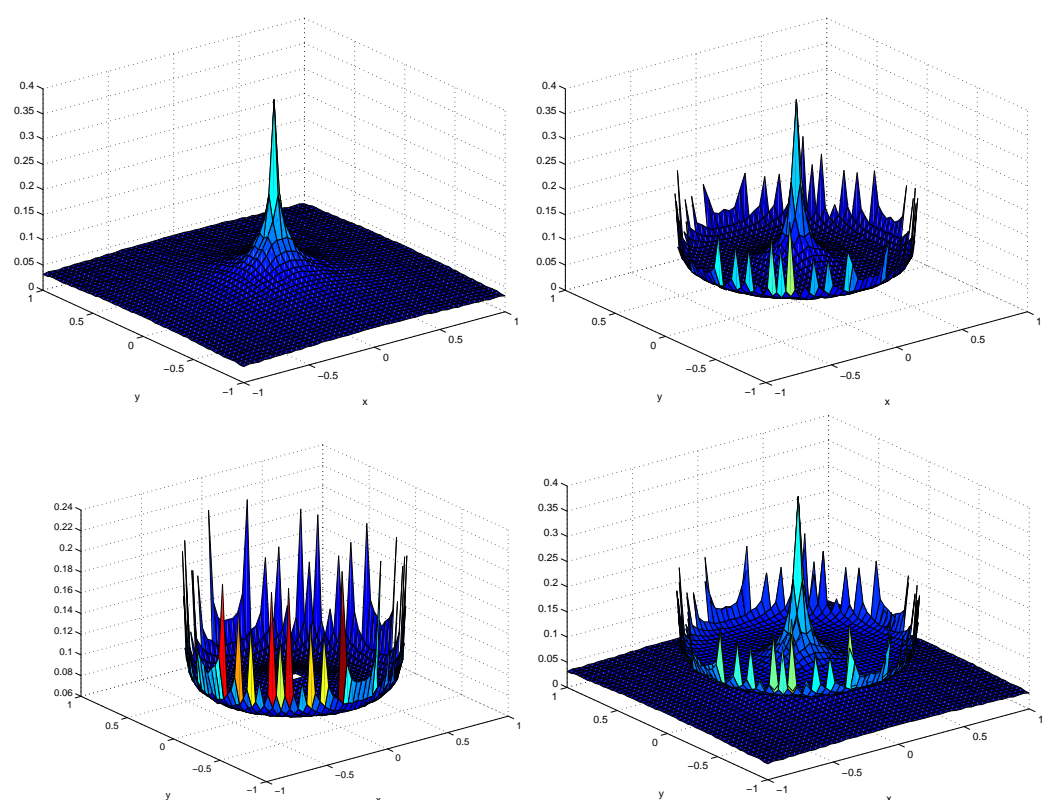

FIG. 5.19. (Example 3) First, second and third arrival amplitudes in physical space at $z=1.5$ using $M=15,1 \leq i, j \leq 51$ and $1 \leq k, l \leq 401$ in phase space; a point source located at $\left(x_{s}, y_{s}\right)=(0,0)$. 


\section{Appendix A. Derivation of equation (3.8).}

The amplitude is transported by

$$
\nabla \cdot\left(A^{2} \nabla \tilde{T}\right)=0 .
$$

Integrating along a ray tube and using the divergence theorem, we get

$$
A \sqrt{\frac{d \sigma}{c}}=A_{0} \sqrt{\frac{d \sigma_{0}}{c_{0}}},
$$

where $\sigma$ is the transverse section area of the ray tube. Furthermore,

$$
A=A_{0} \sqrt{\frac{c d \sigma_{0}}{c_{0} d \sigma}} .
$$

For a point source in an isotropic medium we suppose that the point source emits a directional spherical wave having a normalized radiation function $1 / 4 \pi$ with unit strength,

$$
A_{0}(\tilde{\Theta}, \tilde{\Psi})=\frac{1}{4 \pi R_{0}},
$$

where $\tilde{\Theta}$ and $\tilde{\Psi}$ are the spherical take-off angles, and $R_{0}$ is the radius of the small sphere centered at the point source.

Then at small distances from the point source,

$$
d \sigma_{0}=R_{0}^{2} \sin \tilde{\Theta} d \tilde{\Theta} d \tilde{\Psi} .
$$

Now consider an elementary ray tube,

$$
d V=d x d y d z=d \sigma d s,
$$

where $d s$ is the differential element of arc length. Furthermore,

$$
\begin{gathered}
d V=\frac{\partial(x, y, z)}{\partial(\tilde{T}, \tilde{\Theta}, \tilde{\Psi})} d \tilde{T} d \tilde{\Theta} d \tilde{\Psi} \\
d s=c d \tilde{T}
\end{gathered}
$$

so

$$
d \sigma=\frac{1}{c} \frac{\partial(x, y, z)}{\partial(\tilde{T}, \tilde{\Theta}, \tilde{\Psi})} d \tilde{\Theta} d \tilde{\Psi} .
$$

Thus,

$$
\tilde{A}(z ; x, y)=\frac{c}{4 \pi \sqrt{c_{0}}} \sqrt{\sin \tilde{\Theta}\left|\frac{\partial(\tilde{T}, \tilde{\Theta}, \tilde{\Psi})}{\partial(x, y, z)}\right|} .
$$


Appendix B. Derivation of equation (3.9).

Now, consider the term

$$
\left|\frac{\partial(\tilde{T}, \tilde{\Theta}, \tilde{\Psi})}{\partial(x, y, z)}\right| .
$$

First, we extend all $\tilde{T}, \tilde{\Theta}$ and $\tilde{\Psi}$ to phase space. Then, we replace the $x, y, z$-derivatives by

$$
\begin{aligned}
& \frac{\partial \tilde{(\tilde{)})}}{\partial x}=(.)_{x}+(.)_{\theta} \frac{\partial \theta}{\partial x}+(.)_{\psi} \frac{\partial \psi}{\partial x} \\
& \frac{\partial(\tilde{)})}{\partial y}=(.)_{y}+(.)_{\theta} \frac{\partial \theta}{\partial y}+(.)_{\psi} \frac{\partial \psi}{\partial y} \\
& \frac{\partial \tilde{(\tilde{)})}}{\partial z}=(.)_{x}+(.)_{\theta} \frac{\partial \theta}{\partial z}+(.)_{\psi} \frac{\partial \psi}{\partial z} .
\end{aligned}
$$

Next, one can expand the determinant and get

$$
\left|\frac{\partial(\tilde{T}, \tilde{\Theta}, \tilde{\Psi})}{\partial(x, y, z)}\right|=\frac{1}{c \sin \theta \cos \psi} \frac{\Delta_{1}}{\Delta_{2}} .
$$

This gives

$$
A=\frac{\sqrt{\sin \Theta}}{4 \pi} \frac{c}{\sqrt{c_{0}}} \sqrt{\frac{1}{c \sin \theta \cos \psi} \frac{\Delta_{1}}{\Delta_{2}}},
$$

where $\Theta$ and $\theta$ are the takeoff angle and the arrival angle of the ray from the point source, respectively.

Appendix C. Derivation of equation (4.8).

$\Delta_{1}$ is given by

$$
\Delta_{1}=\left|\begin{array}{cccc}
\phi_{x}^{1} & \phi_{x}^{2} & \Theta_{x} & \Psi_{x} \\
\phi_{y}^{1} & \phi_{y}^{2} & \Theta_{y} & \Psi_{y} \\
\phi_{\theta}^{1} & \phi_{\theta}^{2} & \Theta_{\theta} & \Psi_{\theta} \\
\phi_{\psi}^{1} & \phi_{\psi}^{2} & \Theta_{\psi} & \Psi_{\psi}
\end{array}\right| .
$$

Differentiating this with respect to $z$, we have

$$
\begin{aligned}
& \left(\Delta_{1}\right)_{z}=\left|\begin{array}{cccc}
\left(\phi_{z}^{1}\right)_{x} & \left(\phi_{z}^{2}\right)_{x} & \left(\Theta_{z}\right)_{x} & \left(\Psi_{z}\right)_{x} \\
\phi_{y}^{1} & \phi_{y}^{2} & \Theta_{y} & \Psi_{y} \\
\phi_{\theta}^{1} & \phi_{\theta}^{2} & \Theta_{\theta} & \Psi_{\theta} \\
\phi_{\psi}^{1} & \phi_{\psi}^{2} & \Theta_{\psi} & \Psi_{\psi}
\end{array}\right|+\left|\begin{array}{cccc}
\phi_{x}^{1} & \phi_{x}^{2} & \Theta_{x} & \Psi_{x} \\
\left(\phi_{z}^{1}\right)_{y} & \left(\phi_{z}^{2}\right)_{y} & \left(\Theta_{z}\right)_{y} & \left(\Psi_{z}\right)_{y} \\
\phi_{\theta}^{1} & \phi_{\theta}^{2} & \Theta_{\theta} & \Psi_{\theta} \\
\phi_{\psi}^{1} & \phi_{\psi}^{2} & \Theta_{\psi} & \Psi_{\psi}
\end{array}\right|+ \\
& \left|\begin{array}{cccc}
\phi_{x}^{1} & \phi_{x}^{2} & \Theta_{x} & \Psi_{x} \\
\phi_{y}^{1} & \phi_{y}^{2} & \Theta_{y} & \Psi_{y} \\
\left(\phi_{z}^{1}\right)_{\theta} & \left(\phi_{z}^{2}\right)_{\theta} & \left(\Theta_{z}\right)_{\theta} & \left(\Psi_{z}\right)_{\theta} \\
\phi_{\psi}^{1} & \phi_{\psi}^{2} & \Theta_{\psi} & \Psi_{\psi}
\end{array}\right|+\left|\begin{array}{cccc}
\phi_{x}^{1} & \phi_{x}^{2} & \Theta_{x} & \Psi_{x} \\
\phi_{y}^{1} & \phi_{y}^{2} & \Theta_{y} & \Psi_{y} \\
\phi_{\theta}^{1} & \phi_{\theta}^{2} & \Theta_{\theta} & \Psi_{\theta} \\
\left(\phi_{z}^{1}\right)_{\psi} & \left(\phi_{z}^{2}\right)_{\psi}\left(\Theta_{z}\right)_{\psi} & \left(\Psi_{z}\right)_{\psi}
\end{array}\right|
\end{aligned}
$$

Using (3.2) and the fact that the take-off angles $\Theta$ and $\Psi$ are constant along the characteristics given by the system (4.2), i.e.

$$
\begin{aligned}
& \Theta_{z}+\mathbf{u} \cdot \nabla \Theta=0 \\
& \Psi_{z}+\mathbf{u} \cdot \nabla \Psi=0,
\end{aligned}
$$


we expand the right hand side of (C.2) and replace all $z$-derivative by $x, y, \theta, \psi$ derivatives. Finally, we can get equation (4.8) with some algebra.

Acknowledgment. This research is supported by ONR Grant \#N00014-02-1-0720.

\section{REFERENCES}

[1] D. Adalsteinsson and J.A. Sethian, A fast level set method for propagating interfaces, J. Comput. Phys., 118, 269-277, 1995.

[2] J. D. Benamou, Direct solution of multi-valued phase-space solutions for Hamilton-Jacobi equations, Comm. Pure Appl. Math., 52, 1443-1475, 1999.

[3] J. D. Benamou, An introduction to Eulerian geometrical optics (1992 - 2002), J. Sci. Comp., 19, 63-93, 2003.

[4] R. Burridge, M. V. de Hoop, D. Miller and C. Spencer, Multiparameter inversion in anisotropic media, Geophys. J. Internat., 134, 757-777, 1998.

[5] L. T. Cheng, H. Liu and S. J. Osher, High frequency wave propagation in Schrödinger equations using the level set method, Comm. Math. Sci., 1, 593-621, 2003.

[6] L. T. Cheng, S. J. Osher and J. Qian, Level set based Eulerian methods for multivalued traveltimes in both isotropic and anisotropic media, In 73rd Ann. Internat. Mtg., Soc. Expl. Geophys., Expanded Abstracts, Soc. Expl. Geophys., Tulsa, OK, 1801-1804, 2003.

[7] J. F. Claerbout, Imaging the Earth's Interior, Blackwell Scientific Pub., Palo Alto, CA, 1984.

[8] B. Engquist and O. Runborg, Computational high frequency wave propagation, in Acta Numerica, Cambridge University Press, Cambridge, United Kingdom, 1-86, 2003.

[9] B. Engquist, O. Runborg and A.-K. Tornberg, High frequency wave propagation by the segment projection method, J. Comp. Phys., 178, 373-390, 2002.

[10] M. Falcone and R. Ferretti, Semi-lagrangian schemes for hamilton-jacobi equations, discrete representation formulae and godunov methods, J. Comput. Phys., 175, 559-575, 2002.

[11] S. Fomel and J. Sethian, Fast phase space computation of multiple traveltimes, Proc. Nat. Aca. Sci., 99, 7329-7334, 2002

[12] S. Geoltrain and J. Brac, Can we image complex structures with first-arrival traveltime, Geophysics, 58, 564-575, 1993

[13] L. Gosse, Using K-branch entropy solutions for multivalued geometric optics computations, J. Comput. Phys., 180, 155-182, 2002.

[14] L. Gosse and P. A. Markowich, Multiphase semiclassical approximations of an electron in a one-dimensional crystalline lattice, preprint, 2003.

[15] S. Gray and W. May, Kirchhoff migration using eikonal equation traveltimes, Geophysics, 59, 810-817, 1994.

[16] S. Jin and X. Li, Multi-phase computations of the semi-classical limit of the Schrödinger equation and related problems, Physica D, 182, 46-85, 2003.

[17] S. Jin, H. Liu, S. Osher and R. Tsai, Computing multivalued physical observables for the semi-classical limit of the schrödinger equation, J. Comput. phys., to appear.

[18] S. Jin and S. Osher, A level set method for the computation of multivalued solutions to quasilinear hyperbolic PDEs and Hamilton-Jacobi equations, Comm. Math. Sci., 1, 575-591, 2003.

[19] B. R. Julian and D. Gubbins, Three-dimensional seismic ray tracing, J. Geophys., 43, 95-113, 1977.

[20] J. B. Keller and R. M. Lewis, Asymptotic methods for partial differential equations: the reduced wave equation and Maxwell's equations, Surveys in Applied Mathematics, 1, 1$82,1995$.

[21] H. Liu, L. T. Cheng and S. Osher, A level set formulation for tracking multivalued solutions of nonlinear first order equations, preprint, Level Set Systems, 2003.

[22] X. Liu, S. Osher and T. Chan, Weighted essentially non-oscillatory schemes, J. Comput. Phys., 115, 200-212, 1994.

[23] V. P. Maslov and M. V. Fedoriuk, Semi-classical approximation in quantum mechanics, D. Reidel Publishing Company, 1981.

[24] C. Min, Local level set methods in high dimension and codimension, preprint, UCLA CAM report, 2003.

[25] S. Operto, S. Xu and G. Lambare, Can we image quantitatively complex models with rays, Geophysics, 65, 1223-1238, 2000.

[26] S. Osher, L.T. Cheng, M. Kang, H. Shim and Y.H. Tsai, Geometric optics in a phase-spacebased level set and eulerian framework, J. Comput. Phys., 179, 622-648, 2002. 
[27] D. Peng, B. Merriman, S. Osher, H. K. Zhao and M. Kang, A PDE-based fast local level set method, J. Comput. Phys., 155, 410-438, 1999.

[28] J. Qian, L. T. Cheng and S. J. Osher, A level set based Eulerian approach for anisotropic wave propagations, Wave Motion, 37, 365-379, 2003.

[29] J. Qian and S. Leung, A local level set method for paraxial multivalued geometric optics, submitted to SIAM J. Sci. Comp., UCLA CAM report 03-60, 2003.

[30] J. Qian and S. Leung, A level set method for paraxial multivalued traveltimes, J. Comp. Phys., 197, 711-736, 2004.

[31] J. Qian and W. W. Symes, Adaptive finite difference method for traveltime and amplitude, Geophysics, 67, 167-176, 2002.

[32] S. J. Ruuth, B. Merriman and S. J. Osher, A fixed grid method for capturing the motion of self-intersecting interfaces and related PDEs, J. Comput. Phys., 151, 836-861, 1999.

[33] C. W. Shu, Essentially non-oscillatory and weighted essentially non-oscillatory schemes for hyperbolic conservation laws, Lecture Notes in Mathematics, Springer, 1697, 325-432, 1998.

[34] J. Steinhoff, M. Fan and L. Wang, A new Eulerian method for the computation of propagating short acoustic and electromagnetic pulses, J. Comput. Phys., 157, 683-706, 2000.

[35] J. Strain, Tree methods for moving interfaces, J. Comput. Phys., 151, 616-648, 1998.

[36] J. Strain, Semi-Lagrangian methods for level set equations, J. Comput. Phys., 151, 498-533, 1999

[37] W. W. Symes, Mathematics of reflection seismology, In Annual Report, The Rice Inversion Project, (http://www.trip.caam.rice.edu/), Rice University, 1995.

[38] W. W. Symes, A slowness matching finite difference method for traveltimes beyond transmission caustics, in 68th Ann. Internat. Mtg., Soc. Expl. Geophys., Expanded Abstracts, 1945-1948, Soc. Expl. Geophys., 1998.

[39] W. W. Symes and J. Qian, A slowness matching Eulerian method for multivalued solutions of eikonal equations, J. Sci. Comp., 19, 501-526, 2003.

[40] V. Vinje, E. Iversen, K. Åstebøl and H. Gjøystdal, Estimation of multivalued arrivals in $3 d$ models using wavefront construction - part 1, Geophys. Prosp., 44, 819-842, 1996.

[41] V. Vinje, E. Iversen and H. Gjystdal, Traveltime and amplitude estimation using wavefront construction, Geophysics, 58, 1157-1166, 1993. 